\title{
Using the glial mitochondrial machinery to prevent multilevel dysfunctions observed in a mouse model of Alzheimer's disease
}

Nadia Rosenberg ${ }^{1}$, Maria Reva ${ }^{4}$, Leonardo Restivo ${ }^{1}$, Marc Briquet ${ }^{1}$, Yann Bernardinelli², AnneBérengère Rocher ${ }^{1}$, Henry Markram ${ }^{4}$, and Jean-Yves Chatton ${ }^{1,3^{*}}$

1 Department of Fundamental Neurosciences, University of Lausanne, 1005 Lausanne, Switzerland; ${ }^{2}$ Neonomia, 1205 Geneva, Switzerland; ${ }^{3}$ Cellular Imaging Facility, University of Lausanne, 1005 Lausanne, Switzerland; ${ }^{4}$ Blue Brain Project (BBP), École poly technique fédérale de Lausanne (EPFL), Campus Biotech, 1202 Geneva, Switzerland

*Corresponding author:

Prof. Jean-Yves Chatton, PhD

Dept. of Fundamental Neurosciences

University of Lausanne

Rue Bugnon 9

$\mathrm{CH}-1005$ Lausanne, Switzerland

Tel. +41-21-692-5106 // E-mail: jean-yves.chatton@unil.ch

\section{Author Contributions:}

Conceptualization: NR, JYC, ABR; Methodology: NR, YB, LR, JYC; Investigation: NR, MR, MB; Visualization: NR, MR, LR; Funding acquisition: ABR, HM, JYC; Project administration: JYC; Supervision: JYC, NR; Writing - original draft: NR, JYC; Writing - review \& editing: JYC, NR, MR, LR, ABR

Competing Interest Statement: The authors declare no competing interests. 


\begin{abstract}
Alzheimer's disease $(A D)$ is becoming increasingly prevalent worldwide. It represents one of greatest medical challenge as no pharmacologic treatments are available today that can slow or stop the disease progression. Over time, hippocampal and cerebral atrophy, a core feature of $A D$, results in an amnestic syndrome, whose severity is linked with the extent of neuron loss. Astrocytes play crucial functions within neuronal circuits. The strategic localization of these glial cells allows them to closely interact with neurons, to provide metabolic and functional support, and to regulate interstitial solute composition. Astrocytes are resilient cells contrary to neurons. In our study, we exploited astrocytes as tools to protect the neuronal function in an AD mouse model. We targeted their mitochondria, central to cellular energy metabolism, in the hippocampus and overexpressed the mitochondrial uncoupling protein UCP4, which improves neuronal survival in vitro. We found that this treatment efficiently prevents hippocampal atrophy and neuronal dendritic architecture alteration in $A D$ mice. It also averts aberrant neuronal excitability and alterations of metabolite levels, both altered in AD. Finally, UCP4 overexpression allowed preserving episodic-like memory in $A D$ mice, which is the end goal of future $A D$ therapies. Overall, our results show that targeting astrocyte and their mitochondria is an effective strategy for rescuing neurons facing AD-related stress at early stages of the disease which bears high translational potential.
\end{abstract}

\title{
Significance Statement
}

The brain is extremely energy demanding and particularly vulnerable to mitochondrial damage, which could be central to several types of brain disorders, including neurodegenerative diseases. In a mouse model of Alzheimer's disease, we show that acting in vivo, by inducing mild uncoupling in mitochondria of astrocytes, is able to prevent hippocampal atrophy, to counter metabolic changes, to maintain dendritic architecture, and to prevent aberrant neuronal excitability. Most importantly, spatial memory impairment was prevented in aged Alzheimer mice. This is particularly significant, as preventing cognitive impairment is obviously the ultimate goal of future Alzheimer's disease therapies. 


\section{Main Text}

\section{Introduction}

Alzheimer's disease (AD) is the most prevalent cause of dementia in elderly people and is characterized by deterioration of memory, cognition and behavior. The number of cases is progressing worldwide in an epidemic fashion as humans tend live increasingly longer (1). However, at this time, none of the medications available can slow down or stop the disease progression.

Hallmarks of $A D$ include several pathological changes such as the presence of amyloid immunoreactive senile plaques and tau immunoreactive neurofibrillary tangles (2), associated with decreased neuronal density and atrophy of brain tissue, notably of the hippocampus, which can be detected in patients by structural imaging techniques such as magnetic resonance imaging (MRI) (3). Over time, this brain atrophy results in an amnestic syndrome, whose severity is linked with the extent of neuron loss.

Astrocytes, one of the main class of glia, play crucial functions within neuronal circuits. They are strategically positioned between neurons and the vasculature. Their cellular processes intermingle and are in contact with most of neuronal elements, including axons, dendrites, and cell body (4). They project endfeet that cover most of the vasculature surface. Astrocytes are crucially involved in supporting neuronal function at the level of energy metabolism and by regulating extracellular levels of potentially excitotoxic neurotransmitter glutamate buildup, and of $\mathrm{K}^{+}$released by active neurons. The active participation of astrocytes in modulating synaptic circuits is well recognized. They express a variety of receptors, ion channels, and transporters that allow them to detect as well as influence neuronal activity (5). By releasing a variety of active molecules, they also participate in the establishment, stabilization, and elimination of synaptic connections.

A characteristics of astrocytes is their lower vulnerability compared to neurons in neurodegenerative disease states (6). While neurons are highly oxidative, astrocytes are mostly glycolytic, able to survive without mitochondrial respiration (7). Thus, astrocytes potentially represent entry points for intervening on vulnerable neurons in the AD pathology, in particular before the appearance of cognitive symptoms.

It is well established that disturbances in glucose metabolism, and mitochondrial impairment are crucial player in the initiation of neuronal pathology in AD (8). A key function of mitochondria is ATP production via oxidative phosphorylation mechanisms, which comes with the production of reactive oxygen species (ROS) as byproducts. The imbalance between the rate of ROS production and its clearance leads to oxidative stress, consistently reported in AD (9). Energy deprivation resulting from decreased oxygen and glucose delivery across blood vessels (8) renders neurons particularly vulnerable. Moreover, changes of the oxidative environment contribute to glucose metabolism 
alteration, often by decreasing the activity of glycolytic enzymes $(8,10)$. In addition to ATP production, mitochondria are crucial for the maintenance of cellular calcium homeostasis (11)..

Uncoupling proteins (UCPs) are proton carriers located in the inner mitochondrial membrane that uncouple respiration from ATP production by partially dissipating the proton gradient generated by the electron transport chain. In vitro studies showed that overexpression of mitochondrial uncoupling protein 4 (UCP4), one of the main brain UCP isoform leads to thorough and complex metabolic reorganization in astrocytes (12). As a result, both ROS and mitochondrial ATP production are decreased in astrocytes, acting as a switch that promotes energy production by glycolysis. Most interestingly, astrocytes overexpressing UCP4 and cultured in close apposition to neurons, were found to increase neuronal survival (12).

Rather than tackling the multifactorial causes of $A D$, we decided to focus on its consequences. We therefore hypothesized that astrocytes could be used as a tool to support the function of neurons facing AD-related stress. To achieve this goal, a viral-vector was engineered to specifically express UCP4 in mitochondria of astrocytes in vivo. The viral vector was injected in both hippocampi of wild-type and $3 \times \mathrm{Tg}-\mathrm{AD}$ mice, a widely studied triple-transgenic mouse model of $A D$ (13), which presents calcium dysregulations (14), plasticity (15) and cognitive impairments at young age (16), before onset of neuropathophysiological features (13).

Remarkably, the treatment was found to be effective at preventing hippocampal atrophy. It also preserved dendritic architecture, prevented the development of aberrant electrophysiological and metabolic profiles. Most importantly the treatment successfully maintained episodic-like memory in AD mice. Thus, the innovative approach described in this study holds promise for the development of therapies to combat the progression of $A D$.

\section{Results}

\section{Selective UCP4 expression in mitochondria of astrocytes.}

Because UCP4 overexpression in astrocytes has neuroprotective effects in vitro (12), we hypothesized that in vivo overexpression of UCP4 would have beneficial effects on neurons in pathological conditions, such as AD. To elevate the levels of UCP4 in astrocytes, we designed adeno-associated viruses (AAV) that specifically target mitochondria of astrocytes, namely a vector expressing mCherry only under the GfaABC(1)D astrocytic promoter, which was used as a control, and a vector expressing mCherry coupled to UCP4 (Fig. 1A). A poly HA-tag was added to the UCP4 sequence for later accurate detection of vector expression by immunohistochemistry. The inserted $2 \mathrm{~A}$ self-cleaving peptide sequence allows to yield the same level of expression of mCherry and UCP4. After testing different AAV capsids, we selected serotype 9 that was found to be most efficient at specifically inducing astrocytic expression in the hippocampus. We injected the viral vectors into four separate groups: two groups of wild-type (WT) and two groups of 3xTg-AD mice. 
These groups are referred to as: WT mCherry (WT), WT mCherry-UCP4 (WT UCP4), 3xTg-AD mCherry (3xTg), and 3xTg-AD mCherry-UCP4 (3xTg UCP4). Thus, mice of all groups were injected either with the control mCherry or the mCherry-UCP4 encoding virus at two-months of age (Fig. 1B), before overt neuropathology, mitochondrial impairment, memory impairment, or synaptic dysfunction (13). Viral vectors were injected in the CA1-CA3 and subicular regions of the hippocampus (Fig. 1C). The atrophy of subicular region is the earliest hippocampal anatomical marker of $A D$ (17). The subiculum receives inputs from $C A 1$, which itself receives inputs from CA3 (18). We therefore hypothesized that preserving the neuronal function of the hippocampal network and subiculum will protect mice against AD-related memory impairment.

Fig. 1C confirms that one-month post injection, mCherry-infected cells are astrocytes by colocalizing with glial fibrillary acidic protein (GFAP) immunostaining. We evaluated mitochondrial expression of UCP4 using HA-tag immunostaining, which colocalized with the mitochondrial protein HSP70 in astrocytes (Fig. 1D). As neurons were found not to be infected (SI Appendix, Fig. S1), we could conclude that the viral vectors enable selective expression of UCP4 in mitochondria of astrocytes. Furthermore, we found that UCP4 was still expressed and colocalized with astrocytic mitochondria in 10 months old mice (Fig. 1E), when magnetic resonance imaging (MRI), behavioral, and electrophysiological experiments were carried out.

\section{Astrocytic UCP4 overexpression counters metabolic alterations in 3xTg-AD hippocampi.}

Mitochondria are often referred to as the powerhouses of the cell as they are essential for energy production and regulation. After ensuring that UCP4 was still expressed in astrocyte mitochondria 10 months post injection, we investigated how the metabolism of 3xTg mice was altered at early stages of the disease and what was the effect of UCP4 overexpression. Multiple pathway targeted metabolite screen of central carbon metabolism (including 520 metabolites combining positive and negative ionization mode) revealed that a set of metabolites depicted in Fig. 2A was significantly upregulated in 3xTg mice compared to both WT groups at 3-4 months of age, when mice were still asymptomatic. Interestingly, the metabolic profile of 3xTg UCP4 hippocampi appeared similar to that of WT groups, consistent with a normalizing effect of the UCP4 treatment (SI Appendix, Fig S2). Moreover, pathway analysis highlighting affected biochemical pathways associated with these metabolic changes, unveiled alterations in the TCA cycle, glycolysis and in the metabolism of amino acids (Fig. 2B). These results indicate that (a) profound metabolic perturbations occur in $3 \times \mathrm{Tg}$ hippocampi before overt AD symptoms, which are prevented in 3xTg UCP4 mice, and (b) that UCP4 overexpression in mitochondria of astrocytes is rapidly effective, as its effects appear already 1-2 months post-injection. 
Hippocampal atrophy and dendritic shrinkage of subicular neurons occurring in $3 \times T g-A D$ mice are prevented by astrocytic UCP4 expression.

Hippocampal atrophy is one of the most validated and extensively used biomarkers in AD (3). To investigate whether it is influenced by astrocytic UCP4 overexpression, we performed MRI and compared hippocampal volumes in each group of mice at 8 months of age. We found that $3 \times \mathrm{Tg}$ mice exhibited significant hippocampal atrophy (-18.6 $\pm 5.5 \%)$ compared to WT groups (Fig. 3A). However, 3xTg UCP4 mice showed similar hippocampi volume as both WT groups and was significantly different from 3xTg- hippocampi (Fig. 3B). These results indicate that UCP4 overexpression prevents hippocampal atrophy.

Loss of dendritic architecture integrity, which contributes to cerebral atrophy, is observed in $A D$ in a wide population of neurons, such as subicular pyramidal cells (19). As the subiculum represents the main output from the hippocampus and because it plays a pivotal role in coding spatial information (20), which is compromised in $A D$, we analyzed the dendritic tree of biocytinfilled subicular neurons. Z-stack fluorescence images of neurons were acquired for reconstruction of their 3D structures (Fig. S3 A), which were subsequently used for Sholl and morphometric analysis (Fig. 3, D-G). We divided basal and apical processes in order to compare the dendritic fields among groups of neurons (Fig. $3 \mathrm{C}$ ). 3xTg neurons displayed different dendritic arborization when compared to both WT groups or to 3xTg UCP4 neurons. This observation was confirmed by Sholl analysis. Indeed, while apical dendrites did not present differences among groups ( $S I$ Appendix, Fig. S3 B and C), basal dendrites of 3xTg mice exhibited a significant decrease in Sholl crossings compared to WT mice, corresponding to a marked reduction of the dendritic basal tree complexity (Fig. 3D). Interestingly, we found that UCP4 treatment of 3xTg mice maintained basal dendritic arborization to WT values (Fig. 3E). A drastic reduction in dendritic length was revealed in 3xTg mice compared to both WT groups. However, this alteration was not observed in 3xTg mice overexpressing UCP4 (Fig. 3G). The same trend was observed when considering the total number of dendritic branches (SI Appendix, Fig. S3 D). Thus, UCP4 overexpression in astrocytes conserves dendritic arborization of $3 \times \mathrm{Tg}$ subicular neurons.

\section{Astrocytic UCP4 overexpression prevents the development of aberrant electrophysiological properties in 3xTg-AD subicular neurons and modeling linking architecture alterations with neuronal excitability.}

The elaborate dendritic geometry of a neuron affects it physiological properties, such as its excitability $(21,22)$. For this reason, we decided to characterize the intrinsic excitability of subicular neurons by performing patch-clamp recordings in $\mathrm{WT}, 3 \times \mathrm{Tg}$ and the respective UCP4-treated groups (Fig. 4A). Resting membrane potential (RMP), membrane threshold to evoke a burst, half width, rheobase, tau, input resistance and sag did not show differences between groups ( $S I$ Appendix, Fig. S4, A-G). However, the membrane capacitance of WT and WT UCP4 neurons was 
found to be significantly higher than in 3xTg mice, while the capacitance in 3xTg UCP4 group was not significantly different from the WT groups (SI Appendix, Fig. S4 H). These results show that the viral vectors did not affect the general electrical properties of subicular neurons and that UCP4 overexpression maintains membrane capacitance of $3 x \mathrm{Tg}$ mouse neurons close to values of WT subicular cells.

Next, we measured the instantaneous frequency of the first action potential (AP) burst (Fig. 4B) in response to each suprathreshold current step injections. Fig. 4C shows that 3xTg mice exhibit a significantly higher burst frequency elicited by current injection steps compared to WT and WT UCP4 mice, pointing to an increased excitability that was prevented in 3xTg UCP4 subicular neurons. Depolarization-induced burst firing in subicular neurons is mediated by low-voltageactivated T-type calcium channels (23), which participate in the increases in intracellular calcium. Cytosolic calcium plays a crucial role in neuronal excitability and limits firing frequency trough SK channel activation. Because SK channels are small-conductance calcium-activated potassium channels underlying after-hyperpolarization potential (AHP) (24), we examined AHP amplitude (Fig. 4B) after a train of bursts and APs generated by current step injections. Amplitude of the spikeevoked AHP was significantly larger in 3xTg neurons compared to WT and WT UCP4 neurons. However, AHP amplitude in 3xTg UCP4 neurons presented the same pattern as both WT groups (Fig. 4D). We hypothesized that if SK channels activity is enhanced in AD mice (15), they should show occlusion upon activation, i.e. a strong AHP increase should be induced in WT neurons but

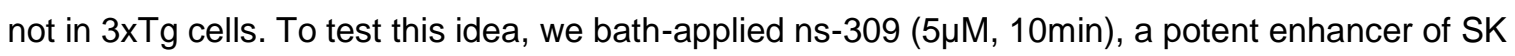
channels (25), which increased AHP in the four groups (Fig. 4E). However, when normalized to the baseline, we found that SK-mediated AHP was significantly increased in WT mice compared to $3 x T g$ mice. Interestingly, upon SK channel activation, 3xTg neurons treated with UCP4 showed an AHP increase comparable to that of WT neurons (Fig. 4F). These data indicate that the higher excitability and activity of SK channels found in 3xTg cells were kept to control levels by astrocytic UCP4 overexpression in 3xTg mice.

To investigate how morphological and electrical alterations shape spike emission and signal propagation after synaptic stimulation, we implemented the 3D morphological reconstructions (Fig. S3 A) and electrophysiological features extracted from patch-clamp recordings (Fig. 4) into full morphology models. The generated models reproduced reliably and accurately experimental data (see SI Appendix, SI Text and SI Appendix, Fig. S5, A-E).

Once validated, we used the models to test the impact of altered dendritic architecture on the output signal of subicular neurons. To do so, 13 or 16 excitatory postsynaptic potentials (EPSPS) were simultaneously injected at different locations of the dendritic tree to elicit somatic spiking (Fig. 4G, middle scheme) in WT model with intact dendritic tree (full morphology) and in models where dendrites were manually shortened by $24 \%$ to match the $3 x T g$ morphometry (reduced morphology). Activation of 13 synapses elicits one spike, which turns into a burst in the reduced morphology (Fig. 
$4 \mathrm{G}$, top left). If more synapses (16) are activated, the voltage response is converted into a burst, whose frequency is increased in the reduced morphology (Fig. 4G, bottom left). The same stimulations were then applied to full and reduced morphologies, with the additional implementation of increased somatic SK channels and dendritic low voltage activated calcium (LVA) conductance ( $\overline{\mathrm{g} S K}, \overline{\mathrm{g}} \mathrm{Ca} \_$LVA) to fit the $3 \times \mathrm{Tg}$ model. Both activation of 13 and 16 synapses induce a burst that is of higher frequency in neurons with shorter dendrites (Fig. 4G, right). These results show that (a) a reduced dendritic arborization increase excitability in WT and WT $\bar{g} S K, \bar{g} C a \_L V A$ models, and $(b)$

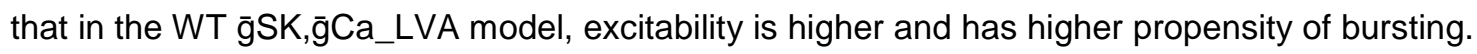

The efficiency of signal propagation from dendrites to soma is a key factor for induction of synaptic plasticity (26), which in turn is crucial for storing engrams in the brain (27). We therefore investigated how voltage signals are propagated along the dendritic branches (Fig. 4H). When stimulated $30 \mu \mathrm{m}$ away from the soma, dendritic and somatic voltages show significantly higher EPSP amplitudes in reduced morphologies, whereas at $60 \mu \mathrm{m}$, only the somatic voltage trace displays a significantly higher amplitude in the cut morphology (Fig. 4I). These results show that a reduced architecture of dendrites as observed in 3xTg mice, alters signal propagation towards the soma providing a causal link between impaired dendritic morphology and increased excitability.

\section{Prevention of spatial memory impairments by UCP4 overexpression in astrocytes.}

Hippocampal-dependent impairment of learning and memory has been linked to aberrant calcium signaling (14) and cellular excitability in AD mice (28). The elevated firing rate of subiculum neurons has been shown to be involved in spatial navigation (29), a cognitive function jeopardized in AD patients. As memory for object location is largely hippocampus-dependent and is impaired in Alzheimer patients at early stages of the disease (30) we performed a novel object location task as depicted in Fig. 5A. During the habituation trial (Fig. 5A, left), mice from all groups traveled an equal distance in the arena, suggesting that viral constructs injected have no effect on the overall locomotory behavior (Fig. S6 A). Next, three objects where placed in fixed locations (Fig. 5A, middle) and mice were allowed to explore them for 5 min during each trial. Mice of all groups show a significant decreased exploration of the objects across sessions (Fig. S6 B), signing the habituation of the exploratory response induced by exposure to novelty. In the last two trials, mice were tested in the novel object location task (Fig. 5A, right), in which one object (object A) was moved to a novel location while the other two objects ( $B$ and $C$ ) remained in their original position. These trials were used to evaluate the innate tendency of rodents to direct exploration towards objects that have been displaced. Lack of reaction to the spatial change has previously been used to assess cognitive impairments in mouse models of Alzheimer's disease (31). The reaction to spatial change was expressed as percent contacts towards the displaced object and compared to the average of percent contacts with the two non-displaced objects. Both WT groups showed a significant reaction to spatial change (Fig. 5, B and D) while mice in the 3xTg group did not exhibit 
exploration levels significantly above the chance level (33.3\%). In contrast, overexpression of UCP4 in 3xTg mice, promoted a significant increase in the exploration directed toward the displaced object (Fig. 5, C and D). These results indicate that uncoupling mitochondria in hippocampal astrocytes via exogenous expression of UCP4 improves reaction to spatial change that is impaired in aging 3xTg mice, in line with the observations shown above of rescued atrophy, dendritic architecture, neuronal excitability, and tissue metabolite profile.

\section{Discussion}

Current $A D$ treatments at best temporarily improve symptoms of memory loss and problems with thinking and reasoning. They are based on molecules that enhance levels of cell-to-cell communication. Other treatment options that have been envisaged include aiming at amyloid $\beta$ plaques, preventing neurofibrillary tangle aggregation, or reducing brain cell inflammation, however so far with limited success (32).

We selected a radically different approach which is of targeting astrocytes within the brain tissue in order to better support neuronal function, which is under AD-stress. Astrocytes offer the key advantage of being more resilient than neurons. Specifically, we targeted mitochondria of astrocytes in vivo, by designing viral vectors with an astrocytic promoter that allow for specific mitochondrial UCP4 expression in hippocampal astrocytes. The expression was found to persist at least until age of 10 months, and showed no sign of adverse effects in WT mice. Remarkably, the astrocytic UCP4 treatment provided a multi-level rescue of AD phenotype ranging from metabolism to spatial memory impairments.

A key question of our study is whether astrocytic overexpression of UCP4 has concrete metabolic effects in vivo at early stages of $A D$. The metabolomic approach that we opted for provided quantitative information on the metabolite level profile in whole hippocampi of the four mice groups studied. It is important to underline that the method does not provide cell type discrimination (astrocytes vs. neurons) nor whether they are located intracellularly or extracellularly. In addition, one does not have the means to distinguish whether an increased level of certain types of metabolites, as we observed in 3xTg mice, results from an upregulation of a specific metabolic pathway, or if it is due to an impairment of catabolic enzymes involved in the pathway. For instance, in the case of lactate found to be significantly elevated in 3xTg mice compared to the other groups, one cannot discriminate if it results from glycolysis upregulation or from impaired neuronal consumption. In the latter scenario, metabolites would not enter the TCA cycle and accumulate in the cell. Regardless of the underlying mechanisms, one can conclude that metabolites of TCA cycle, pyruvate metabolism, glycolysis, and histidine metabolism are found in higher concentrations in 3xTg mice compared to both WT groups. These observations are consistent with a recent study (33) showing higher concentrations in both plasma and cerebrospinal fluid of TCA cycle 
intermediates in AD patients compared to control subjects. In line with our results, another study showed that metabolites of glycolysis such as phosphoenolpyruvate, among others, are increased in 3xTg male mice hippocampus compared to non-transgenic mice at 3 months of age (34). Our analysis showed the striking result that, while several classes of metabolites involved in energygenerating pathways are increased in 3xTg mice, they are maintained to basal levels in 3xTg mice overexpressing astrocytic UCP4. One could postulate that the beneficial effects of the UCP4 treatment starting early on during treatment underlies the rescue of several $A D$ phenotypes (hippocampal and dendritic atrophy, neuronal physiology, and behavior) that we observed later at 7-10 months of age.

The first notable observation was that the UCP4 treatment prevented the development of hippocampal atrophy in 3xTg-AD mice, as quantified using MRI, an imaging approach routinely used with AD patients (3). At the microscopic level, neurons of the subiculum, the main outputs of the hippocampus, displayed reduced arborization in 3xTg mice as observed in different AD mouse models $(21,35)$. Atrophy of dendritic tree contributes to tissue volume loss. Interestingly, the dendritic architecture was preserved in UCP4-treated mice. These morphological observations made on 7-10 months old mice were an important indication that the approach was favorably acting on 3xTg-AD brains.

The next phase was to investigate whether there was a measurable effect of the treatment on the physiology of neurons in the pathological stage (7-10 months). We found an increased excitability in 3xTg-AD subicular neurons that was translated into an increase in AHP amplitude and burst fire frequency. A larger AHP could arise from exaggerated calcium release from the endoplasmic reticulum, for instance resulting from the upregulation of ryanodine receptors as described in 3xTg-AD mice (36). Moreover, it has been shown that small-conductance calciumactivated SK2 channel activity is increased in CA1 neurons of 3xTg-AD mice (15), along with the coupling between SK channels and ryanodine receptors (36). We found evidence by enhancing SK channel activity, that the increase in AHP, in addition to be likely exacerbated by intracellular calcium, also results from an enhancement of SK channel activation. As SK protein levels have been reported to be unchanged in $A D$ mice (15), its increased activation could arise from a change in biophysical conformation increasing the conductance of the channel as suggested by our full morphology computational modeling. Another explanation for the increase in burst frequency derives from dendritic architecture alteration (21) that we measured in subicular neurons of $3 \times \mathrm{Tg}$ AD mice. While in WT neuronal models the reduced morphology of dendrites caused the transition from single spike to bursting, in the WT model with increased SK and LVA conductance, simulating $3 x \operatorname{Tg}-A D$ mice, it increased burst firing frequency, which supports our electrophysiological data. In shorter dendrites, the signal induced by synaptic stimulations can propagate more efficiently towards the axosomatic compartment without being diluted by backpropagation in more distal dendrites (37). These changes in excitability counterbalanced by astrocytic UCP4 overexpression, 
could contribute to synaptic plasticity alterations and subsequent AD-related network dysfunction, resulting in cognitive impairments.

Obviously, the end goal of $A D$ treatments is to alleviate cognitive impairments. Progressive memory loss is the hallmark of $A D$ and is among the first symptoms reported by patients and their families. At first, only short-term memory is impaired followed by fragmentation of long termmemory. In early stages of $A D$, patients often suffer from spatial memory impairment, such as spatial disorientation, and tend to forget recently acquired information, e.g. fail to remember the location of an object soon after seeing it. This behavior involves spatial working memory, a type of short-term memory that can be addressed in rodents with an object spatial recognition task (38). Our results show that $3 \times \mathrm{Tg}$ mice did not spend as much time as WT mice exploring the object that was shifted to a novel location, demonstrating compromised cognitive performances (31). The astrocytic overexpression of UCP4 allowed $3 \times \mathrm{Tg}$ mice to maintain a normal exploring behavior towards the object found in the novel location. It is generally accepted that the pattern of memory impairments in patients correlate with parameters defining both structural and functional brain integrity. As spatial memory is hippocampus-dependent (39), this statement fits our data that show correlation between hippocampal atrophy and poor spatial memory performance of $3 \times \mathrm{Tg}$ mice and, conversely, normal memory performance when structural alteration of the hippocampus is prevented as seen in mice overexpressing astrocytic UCP4.

Finally, while not directly addressing how UCP4 overexpression in astrocytic mitochondria may favorably act on neurons in the context of $A D$, one could formulate some working hypotheses. Firstly, the UCP4 treatment had a normalizing effect on a range of metabolites very early in the hippocampus of 3xTg-AD mice. This favorable effect on metabolite levels could enable 3xTg-AD neurons to maintain physiological functioning, avoiding the buildup of oxidative stress linked with altered metabolism. It may also prevent the appearance of unbalanced expression levels of key enzymes of the metabolism.

Secondly, increased levels of UCP4 in neurons was shown to enhance their resistance to metabolic and oxidative stress (40). In parallel, a study demonstrated that astrocytes release and transfer functional mitochondria to neurons, promoting their survival and plasticity after stroke in a CD38 signaling-dependent manner (41). Brain CD38 is mainly expressed in glia and its expression in astrocytes was shown to be enhanced by neuronally-released glutamate (42). Because hyperexcitability observed in 3xTg-AD mice could contribute to increased glutamate release in the extracellular space, mitochondrial transfer from astrocytes to neurons could represent a plausible rescue-mechanism providing early support to neurons facing AD-related injury. Further evaluations are undoubtedly needed to decipher the mechanisms behind the multi-level rescue provided by astrocytic UCP4 overexpression. 
In conclusion, we propose that targeting astrocytes and their mitochondria is an innovative strategy to preserve cellular and cognitive functions in $A D$ and has strong potential for the development of efficacious gene therapies for $A D$ and potentially for other brain pathologies.

\section{Materials and Methods}

\section{Animals and viral vectors.}

All the experimental procedures complied with the Swiss National Institutional Guidelines on animal experimentation and were approved by the canton of Vaud Cantonal Veterinary Office Committee for Animal Experimentation (authorization \#VD3106 and VD3106_1). Details on the viral vector construction, surgery, and stereotaxic viral vector delivery, can be found in SI appendix, Materials and Methods.

\section{Behavioral assay.}

Novel Object Location testing was performed using an arena which contained three objects with geometric shapes. The experiment consisted of 6 trials run in one single day. In the novel object location trials, one object was moved and the reaction to spatial change was defined as the total number of contacts with the displaced object expressed as percent contacts over the total number of contacts with the 3 objects. For details of the behavioral experiments, see SI appendix, Materials and Methods.

\section{Acute slice preparation and patch clamp recordings.}

Acute brain slices were prepared for whole cell patch-clamp recordings of neurons of the subiculum. For details of the procedures, see SI appendix, Materials and Methods.

\section{Electrophysiological modeling.}

To construct detailed neuronal models based on the electrophysiological recordings we first extracted features from the available voltage recordings. Then, the sets of ionic mechanisms present in the cell were defined and combined with 3D morphological reconstructions. We optimized maximal conductance of ionic mechanisms, such that the responses of the model reproduce features extracted from experiments. Details on the modeling approach are found in $S I$ Appendix, SI Materials and Methods.

\section{Metabolomics profiling.}

Steps performed to assess metabolite profiling such as microwave brain fixation, metabolite extraction, protein quantification, multiple pathway targeted analysis, quality control, data processing, and statistical analyses of the metabolomics profiling, are found in detail in $\mathbf{S I}$ Appendix, SI Materials and Methods. Pathway analysis was performed using MetaboAnalyst 5.0 
(https://www.metaboanalyst.ca/).

\section{Statistics and data analysis.}

The statistical tests used are listed in the respective Figure legends. Graphs and statistical analyses were performed with GraphPad Prism.

\section{Acknowledgments}

This research was supported by the Synapsis Foundation Alzheimer Research Switzerland (JYC), and by the Blue Brain Project, a research center of the École polytechnique fédérale de Lausanne, EPFL, from the Swiss government's ETH Board of the Swiss Federal Institutes of Technology (HM, MR). We thank A. Benechet and R. Colotti (IVIF, Unil-CHUV) for their help with MRI experiments. A. Arnaudon (BBP) for assistance with morphometrics packages. W. Van Geit (BBP) for the supervisory support. We thank J. Ivanisevic and colleagues of the Metabolomics Platform of the Faculty of Biology and Medicine at University of Lausanne for their expert handling of the metabolomic profiling. The behavioral experiments were run in the behavioral core of the Department of Fundamental Neuroscience, University of Lausanne (Neuro-Behavioral Analysis Unit, NeuroBau).

\section{References}

1. Anonymous, 2021 Alzheimer's disease facts and figures. Alzheimers Dement. 17, 327-406 (2021).

2. H. Braak, E. Braak, Neuropathological stageing of Alzheimer-related changes. Acta Neuropathol. 82, 239-259 (1991).

3. L. G. Apostolova et al., Conversion of mild cognitive impairment to Alzheimer disease predicted by hippocampal atrophy maps. Arch. Neurol. 63, 693-699 (2006).

4. A. Verkhratsky, M. Nedergaard, Physiology of Astroglia. Physiol. Rev. 98, 239-389 (2018).

5. M. Santello, N. Toni, A. Volterra, Astrocyte function from information processing to cognition and cognitive impairment. Nat. Neurosci. 22, 154-166 (2019).

6. S. Saxena, P. Caroni, Selective neuronal vulnerability in neurodegenerative diseases: from stressor thresholds to degeneration. Neuron 71, 35-48 (2011).

7. L. M. Supplie et al., Respiration-Deficient Astrocytes Survive As Glycolytic Cells In Vivo. J. Neurosci. 37, 4231-4242 (2017).

8. L. Mosconi, A. Pupi, M. J. De Leon, Brain glucose hypometabolism and oxidative stress in preclinical Alzheimer's disease. Ann. N. Y. Acad. Sci. 1147, 180-195 (2008).

9. X. Wang et al., Oxidative stress and mitochondrial dysfunction in Alzheimer's disease. Biochim. Biophys. Acta 1842, 1240-1247 (2014). 
10. D. A. Butterfield, B. Halliwell, Oxidative stress, dysfunctional glucose metabolism and Alzheimer disease. Nat. Rev. Neurosci. 20, 148-160 (2019).

11. L. Contreras, I. Drago, E. Zampese, T. Pozzan, Mitochondria: the calcium connection. Biochim. Biophys. Acta 1797, 607-618 (2010).

12. H. Perreten Lambert et al., Control of mitochondrial $\mathrm{pH}$ by uncoupling protein 4 in astrocytes promotes neuronal survival. J. Biol. Chem. 289, 31014-31028 (2014).

13. S. Oddo et al., Triple-transgenic model of Alzheimer's disease with plaques and tangles: intracellular Abeta and synaptic dysfunction. Neuron 39, 409-421 (2003).

14. S. Chakroborty, I. Goussakov, M. B. Miller, G. E. Stutzmann, Deviant ryanodine receptormediated calcium release resets synaptic homeostasis in presymptomatic 3xTg-AD mice. $J$. Neurosci. 29, 9458-9470 (2009).

15. S. Chakroborty et al., Early presynaptic and postsynaptic calcium signaling abnormalities mask underlying synaptic depression in presymptomatic Alzheimer's disease mice. J. Neurosci. 32, 8341-8353 (2012).

16. L. K. Clinton et al., Age-dependent sexual dimorphism in cognition and stress response in the 3xTg-AD mice. Neurobiol. Dis. 28, 76-82 (2007).

17. G. A. Carlesimo et al., Atrophy of presubiculum and subiculum is the earliest hippocampal anatomical marker of Alzheimer's disease. Alzheimers Dement. (Amst) 1, 24-32 (2015).

18. N. M. van Strien, N. L. Cappaert, M. P. Witter, The anatomy of memory: an interactive overview of the parahippocampal-hippocampal network. Nat. Rev. Neurosci. 10, 272-282 (2009).

19. D. G. Flood, Region-specific stability of dendritic extent in normal human aging and regression in Alzheimer's disease. II. Subiculum. Brain Res. 540, 83-95 (1991).

20. S. M. Kim, S. Ganguli, L. M. Frank, Spatial information outflow from the hippocampal circuit: distributed spatial coding and phase precession in the subiculum. J. Neurosci. 32, 1153911558 (2012).

21. Z. Šišková et al., Dendritic structural degeneration is functionally linked to cellular hyperexcitability in a mouse model of Alzheimer's disease. Neuron 84, 1023-1033 (2014).

22. W. Rall, Theory of physiological properties of dendrites. Ann. N. Y. Acad. Sci. 96, 1071-1092 (1962).

23. S. M. Joksimovic et al., The role of T-type calcium channels in the subiculum: to burst or not to burst? J. Physiol. 595, 6327-6348 (2017).

24. M. Stocker, M. Krause, P. Pedarzani, An apamin-sensitive $\mathrm{Ca2+-activated} \mathrm{K}+$ current in hippocampal pyramidal neurons. Proc. Natl. Acad. Sci. U.S.A. 96, 4662-4667 (1999).

25. Y. W. Nam et al., Structural insights into the potency of SK channel positive modulators. Sci. Rep. 7, 17178 (2017).

26. G. J. Stuart, N. Spruston, Dendritic integration: 60 years of progress. Nat. Neurosci. 18, 17131721 (2015). 
27. T. Takeuchi, A. J. Duszkiewicz, R. G. Morris, The synaptic plasticity and memory hypothesis: encoding, storage and persistence. Philos. Trans. R. Soc. Lond. B Biol. Sci. 369, 20130288 (2014).

28. K. E. Davis, S. Fox, J. Gigg, Increased hippocampal excitability in the 3xTgAD mouse model for Alzheimer's disease in vivo. PLoS One 9, e91203 (2014).

29. T. Kitanishi, R. Umaba, K. Mizuseki, Robust information routing by dorsal subiculum neurons. Sci. Adv. 7 (2021).

30. R. P. Kessels, S. Rijken, L. W. Joosten-Weyn Banningh, V. A. N. E. N. Van Schuylenborgh, M. G. Olde Rikkert, Categorical spatial memory in patients with mild cognitive impairment and Alzheimer dementia: positional versus object-location recall. J. Int. Neuropsychol. Soc. 16, 200-204 (2010).

31. S. D. Creighton et al., Dissociable cognitive impairments in two strains of transgenic Alzheimer's disease mice revealed by a battery of object-based tests. Sci. Rep. 9, 57 (2019).

32. J. Cummings, Correction to: New approaches to symptomatic treatments for Alzheimer's disease. Mol. Neurodegener. 16, 21 (2021).

33. V. van der Velpen et al., Systemic and central nervous system metabolic alterations in Alzheimer's disease. Alzheimers Res. Ther. 11, 93 (2019).

34. Y. Dong, G. J. Brewer, Global Metabolic Shifts in Age and Alzheimer's Disease Mouse Brains Pivot at NAD+/NADH Redox Sites. J. Alzheimers Dis. 71, 119-140 (2019).

35. D. L. Moolman, O. V. Vitolo, J. P. Vonsattel, M. L. Shelanski, Dendrite and dendritic spine alterations in Alzheimer models. J. Neurocytol. 33, 377-387 (2004).

36. G. E. Stutzmann et al., Enhanced ryanodine receptor recruitment contributes to $\mathrm{Ca2+}$ disruptions in young, adult, and aged Alzheimer's disease mice. J. Neurosci. 26, 5180-5189 (2006).

37. G. Stuart, N. Spruston, B. Sakmann, M. Häusser, Action potential initiation and backpropagation in neurons of the mammalian CNS. Trends Neurosci. 20, 125-131 (1997).

38. E. Dere, J. P. Huston, M. A. De Souza Silva, Integrated memory for objects, places, and temporal order: evidence for episodic-like memory in mice. Neurobiol. Learn. Mem. 84, 214221 (2005).

39. N. J. Broadbent, L. R. Squire, R. E. Clark, Spatial memory, recognition memory, and the hippocampus. Proc. Natl. Acad. Sci. U.S.A. 101, 14515-14520 (2004).

40. D. Liu et al., Mitochondrial UCP4 mediates an adaptive shift in energy metabolism and increases the resistance of neurons to metabolic and oxidative stress. Neuromolecular Med. 8, 389-414 (2006).

41. K. Hayakawa et al., Transfer of mitochondria from astrocytes to neurons after stroke. Nature 535, 551-555 (2016).

42. S. Bruzzone et al., Glutamate-mediated overexpression of CD38 in astrocytes cultured with neurones. J. Neurochem. 89, 264-272 (2004). 


\section{Figures}

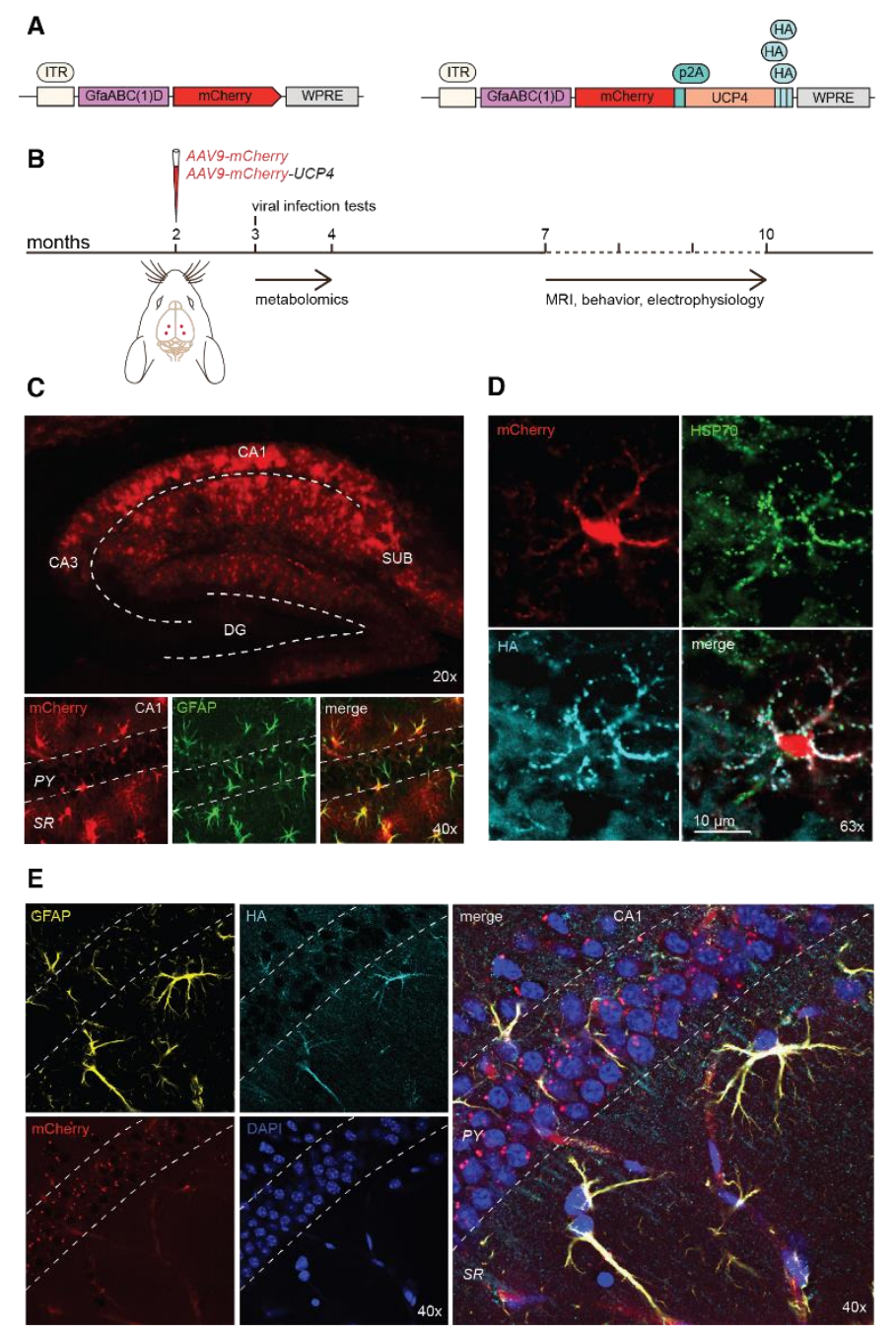

Fig. 1. Characterization and validation of viral vectors used to drive UCP4 expression in astrocytes of the hippocampus. (A) Design of AAV vectors used to infect astrocytes. Left: construct containing mCherry alone under astrocytic promoter (GfaABC(1)D) used as a control. Right: Vector containing mCherry coupled to UCP4, which contains a poly-HA tail. (B) Timeline of experimental procedures. Two-months old WT and 3xTg-AD mice were injected bilaterally in the hippocampus with the AAV9mCherry or the AAV9-mCherry-UCP4 viral vectors (red dots: injection sites). Animals were tested starting at 7 months of age. (C) Confocal images showing mCherry expression (red), GFAP staining (green) and the merged image in the hippocampus one month after viral infection (DG; dentate gyrus, SUB: subiculum). Images show that mCherry colocalizes with GFAP positive astrocytes (green). (D) Immunostaining of the mitochondrial marker HSP70 (green) and UCP4 HA-tag (cyan) plus mCherry expression (red) and the merged image, showing that tagged UCP4 is expressed in mitochondria of astrocytes. (E) Immunostaining of GFAP (yellow) and HA-tag (cyan) plus mCherry indicates that tagged UCP4 is still expressed 10 months post-injection and remains present in astrocytes. DAPI staining (blue) was used to visualize the CA1 pyramidal layer. ( $P Y$ : pyramidal layer, $S R$ : stratum radiatum). 


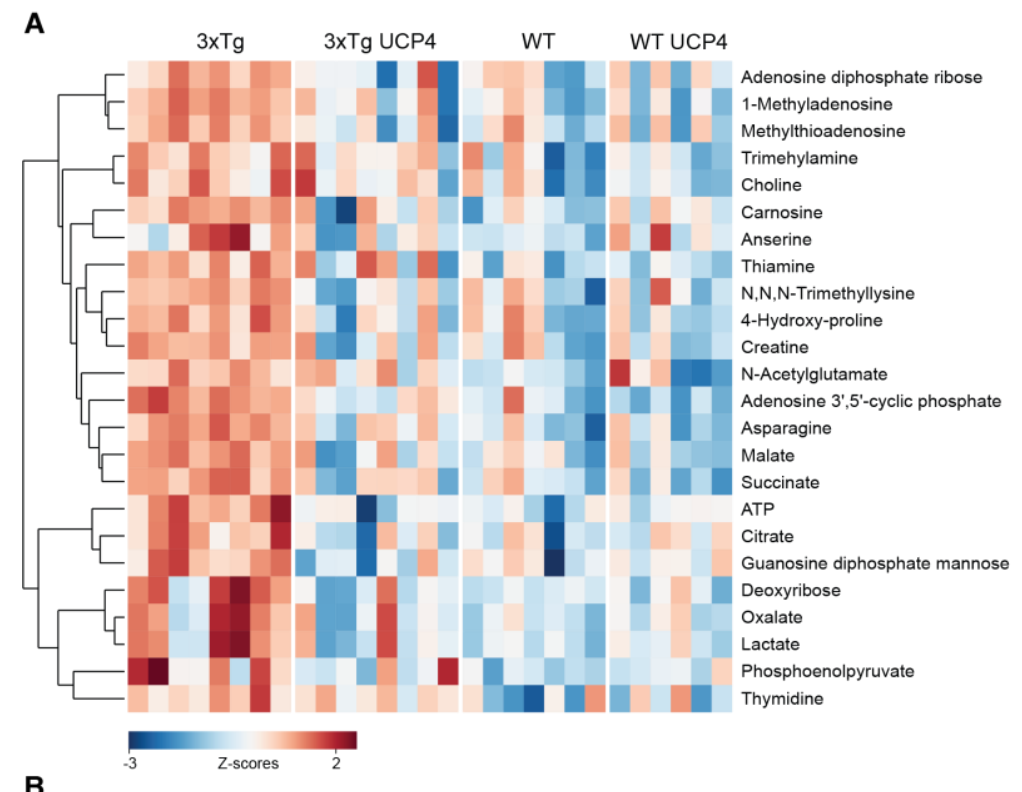

B

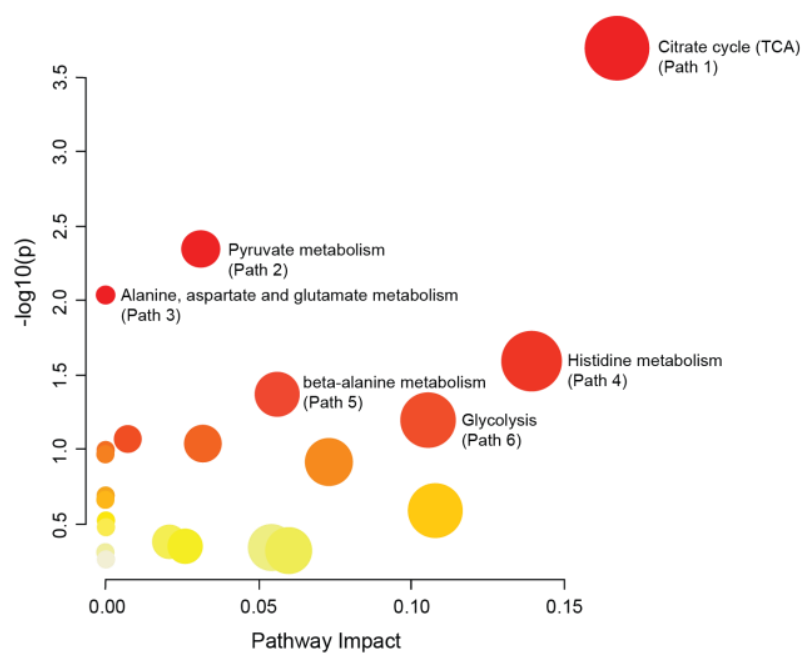

Fig. 2. Astrocytic UCP4 overexpression maintains normal hippocampal metabolite profile in 3xTgAD mice. (A) Heat map of metabolites whose levels changed significantly in 3xTg mice compared to both WT groups and 3xTg UCP4 mice (see also Fig. S2). Each row represents a metabolite and each column a different mouse. (B) Pathway analysis performed on metabolomics results showed in (A). Each node represents an altered metabolic pathway in the hippocampus of 3xTg mice that is preserved in 3xTg UCP4 mice and their size indicates the impact of this pathway. 
A

A

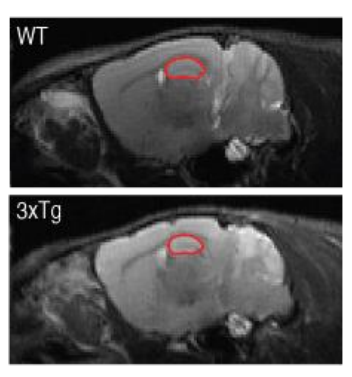

B

C
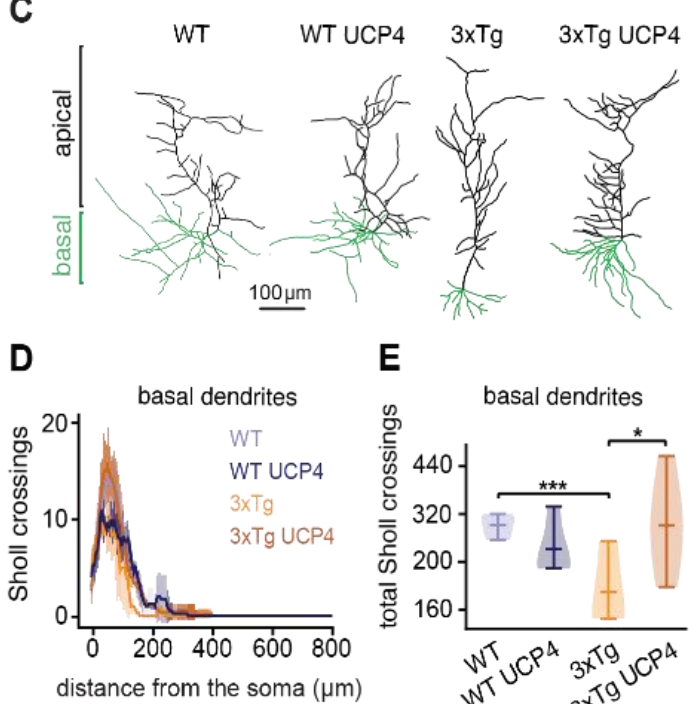

E
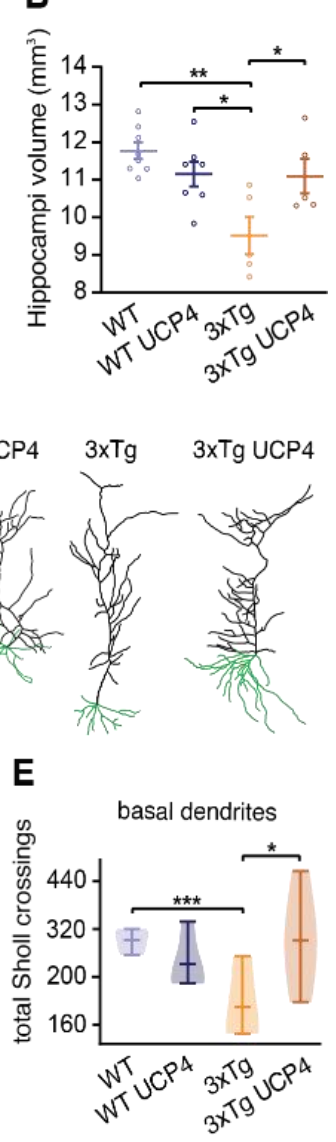

$\mathbf{F}$

G
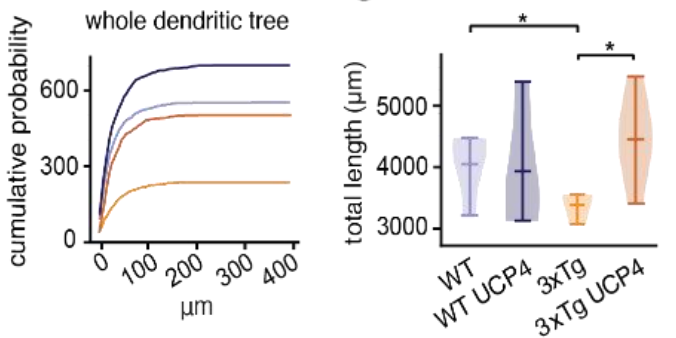

Fig. 3. Hippocampal atrophy and alteration of dendritic arborization of 3xTg-AD neurons is impeded by UCP4 overexpression. (A) In vivo MRI of mice hippocampus. Representative brain T2-weighted images of a WT and a 3xTg mouse in sagittal plane. Red dashed lines indicate ROI for the hippocampus. (B) Quantification of hippocampal volume (WT: $n=8$, WT UCP4: $n=7,3 x T g: n=5$, $3 \times \operatorname{Tg}$ UCP4: $n=5$ ). Each circle represents the volume of combined left and right hippocampus per mouse. (C) Examples of reconstructed morphologies from the 3D models with identified apical (black) and basal (green) dendrites. (D) Sholl profiles of basal dendrites, calculated using $5 \mu \mathrm{m}$ increments (WT: $n=4$, WT UCP4: $n=4,3 \times T g: n=3$, 3xTg UCP4: $n=5$ ). (E) Total number of Sholl crossings for each group. (nonparametric bootstrapping test, $3 \times \mathrm{Tg}$ vs WT: $P=0.001$, 3xTg vs $3 \times \mathrm{Tg}$ UCP4: $P=0.03)$. (F) Cumulative probability plot of total dendritic length $(\mu \mathrm{m})$ for each group. $(\mathbf{G})$ Total path length of dendritic tree for each group calculated per morphology. In (B) one-way ANOVA followed by Tukey's post hoc test was performed. Error bars in this figure are mean \pm SEM. ${ }^{\star} P<0.05,{ }^{* *} P<0.01$. In (E and $\left.\mathbf{G}\right)$ nonparametric bootstrapping test was performed, ${ }^{\star} P<0.05$, ${ }^{* * *} P<0.001$. Bars on violin plots: mean $\pm \mathrm{Cl}$. 

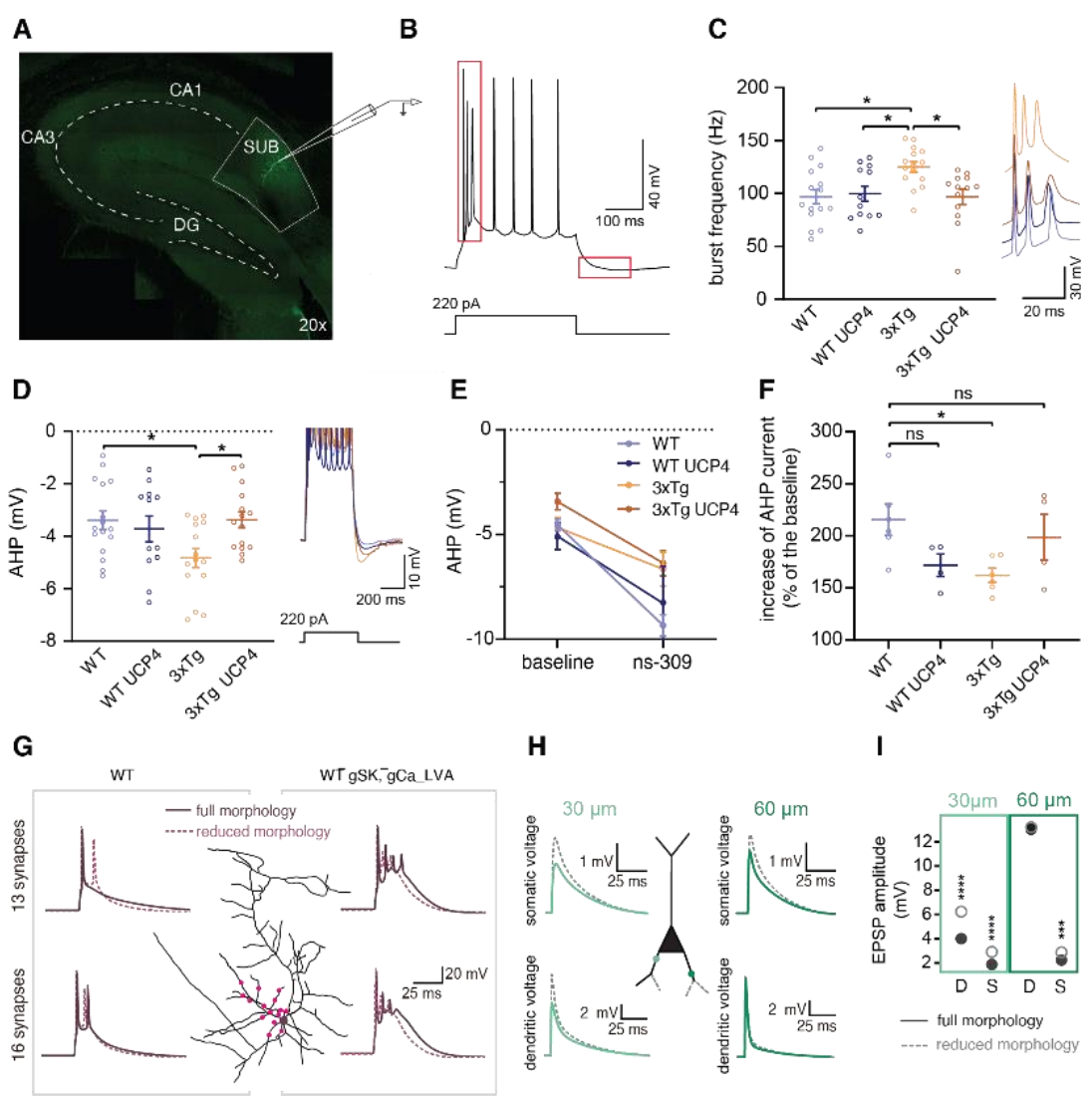

H

I
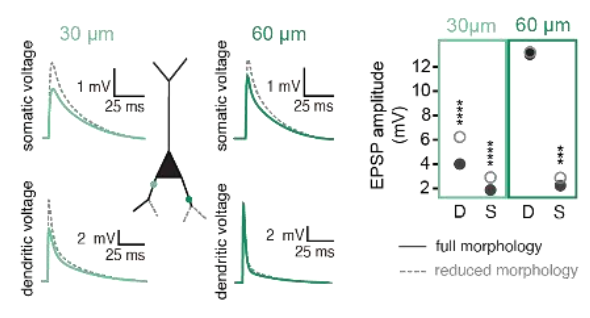

Fig. 4. Hyperexcitability in 3xTg-AD subicular neurons is hampered by UCP4 overexpression and modeling makes the link between dendritic morphology and neuronal excitabiliy. (A) Confocal image of the hippocampus and the area of patch-clamp recordings highlighted by a white line (DG: Dentate gyrus, SUB: subiculum). (B) Example trace of a current-clamp recording. Red rectangles represent the temporal windows used to analyze the first burst frequency and the amplitude of AHP ( $\mathbf{C}$ and $\mathbf{D}$, respectively). (C) Plot of fire frequency of the first burst. Symbols represents the average of all the sweeps per cell. (WT: $n=15$, WT UCP4: $n=12$, 3xTg: $n=15$, 3xTg UCP4: $n=13$ ). Inset: representative traces of the first burst occurring after depolarization. (D) Amplitude of the spikeevoked AHP. Symbols represent the average AHP of all the sweeps per cell. (WT: $n=16$, WT UCP4: $n=12$, 3xTg: $n=15$, 3xTg UCP4: $n=15$ ). Inset: representative spike trains and subsequent AHP. (E) AHP amplitude in control condition and after SK channel activation using ns-309. Symbols represent the average of all mice. (WT: $n=6, W T$ UCP $4: n=4,3 x T g: n=6,3 x T g$ UCP4: $n=4$ ). (F) Plot showing the ns-309 mediated enhancement of AHP amplitude as a percentage of the baseline. Each circle represents one neuron (same $\mathrm{n}$ as in $\mathbf{E}$ ). (G) Center: illustration of stimulation sites at different locations along the dendritic tree (pink dots) to elicit somatic spike in the soma (purple dot). Left: voltage traces when 13 EPSPs (top) or 16 EPSPs (bottom) are simultaneously injected in WT condition. Right: voltage traces when 13 EPSPs or 16 EPSPs are simultaneously injected in simulated increased somatic $\bar{g} S K$ and dendritic $\bar{g}$ Ca_LVA. (H) Effect of morphology on signal propagation. Voltage traces in the soma (top traces) and in the dendrite (bottom traces) after EPSP stimulation at $30 \mu \mathrm{m}$ (light green rectangle) and 60 $\mu \mathrm{m}$ (dark green rectangle) from the soma. In G and $\mathbf{H}$ Solid line: full WT dendritic length, dashed line: shortened dendritic tree that matches the 3xTg morphometrics analysis. (I) Quantification of panel $\mathbf{H}$ results: voltage amplitudes in dendrite (D) and soma (S) followed by EPSP stimulation for 4 WT models (same as in Fig.3). Dendritic and 
bioRxiv preprint doi: https://doi.org/10.1101/2022.01.25.477694; this version posted January 27, 2022. The copyright holder for this preprint (which was not certified by peer review) is the author/funder, who has granted bioRxiv a license to display the preprint in perpetuity. It is made available under aCC-BY-NC-ND 4.0 International license.

somatic amplitudes are statistically different in case of proximal EPSP stimulation (bootstrap permutation test, dendritic: $P=0$; somatic: $P=0$ ), while for distant stimulation statistical difference is only detected in somatic voltage. In (C), (D) and (F) one-way ANOVA followed by Tukey's post hoc test was performed. Error bars in this figure are mean \pm SEM. ${ }^{*} P<0.05$, ns; not significant. In (I), bootstrap permutation test was performed. ${ }^{* \star *} P<0.001$; ${ }^{* \star *} P<0.0001$. Bars on violin plots: mean $\pm \mathrm{Cl}$. 
A
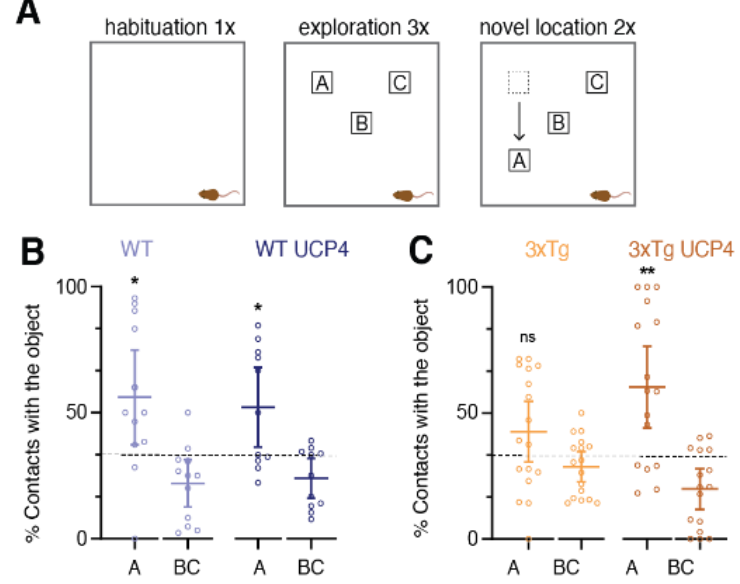

D
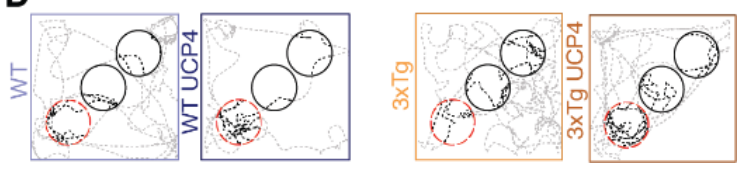

Fig. 5. Mild mitochondrial uncoupling of astrocytes prevents spatial memory impairments of 3xTgAD mice. (A) Schematic representation of the spatial recognition task. (WT: $n=12$, WT UCP4: 11, 3xTg: $n=18,3 x \operatorname{Tg} \cup C P 4: \mathrm{n}=16$ ). (B) and (C) Plots showing the reaction to spatial change expressed as the \% of contact with the objects. $A$ is the displaced object and BC is the average of the two objects that were not moved. Each symbol represents a different mouse. The dashed line represents the chance level (33.3\%). (D) Representative tracking plots during the NOL trials. Black circles indicate the OEZ containing the objects that have not been moved ( $B$ and $C$ ). Red dashed circle shows the novel location zone of object $A$. Black dashed line represents the path followed by the mouse. In (B) one-way ANOVA followed by Tukey's post hoc test was performed. In (B) and (C) one sample t test was performed. Error bars are mean \pm Cl. ${ }^{*} P<0.05 ;{ }^{* * *} P<0.0001$. 
bioRxiv preprint doi: https://doi.org/10.1101/2022.01.25.477694; this version posted January 27, 2022. The copyright holder for this preprint (which was not certified by peer review) is the author/funder, who has granted bioRxiv a license to display the preprint in perpetuity. It is made available under aCC-BY-NC-ND 4.0 International license.

\section{Supplementary Text}

\section{Computational modeling of neuronal electrical properties.}

Ionic mechanisms present in cells were defined and optimized such that the responses of the models reproduce features extracted from experiments (fig. S5 A). After model optimization for each of the four groups, simulations of 200,300 and 450 pA current injections were produced to test how burst frequency and AHP would behave in the models. Current injections led to statistical differences between $3 \times \mathrm{Tg}$ and the other groups translated into an increase in burst frequency and AHP (fig. S5, B-C), as was observed in experimental data (Fig. 4, C-D). To simulate ex-vivo conditions of SK channels activation with bath applied ns-309, SK channel conductance ( $\bar{g} S K)$ was manually increased and pooled across responses of the models to 200 and $450 \mathrm{pA}$ current injections. We observed that in all groups the simulated $\bar{g} S K$ increase enhanced AHP compared to the baseline (fig. S5 D). The relative AHP increase was however significantly smaller in $3 \times \mathrm{Tg}$ models compared to other groups (fig. S5 E), as previously observed in electrophysiological recordings (Fig. 4F). Thus, the generated models accurately reproduce experimental data.

SK channels play crucial role notably in intrinsic excitability and in shaping postsynaptic responses (1). Their activity is increased in 3xTg mice (2). SK channels are voltage-independent channels, solely activated by elevations of intracellular calcium concentrations that may derive from different sources (3). To identify key elements underlying prevention of aberrant burst frequency and AHP by UCP4 overexpression, we selected a series of model parameters based on their relevance for cell excitability and SK channel activity. Those include unitary capacitance $(\mathrm{cm})$, sodium channel conductance $(\overline{\mathrm{g} N a})$, persistent potassium conductance ( $\bar{g} \mathrm{~K} \_$Pst), transient potassium conductance ( $\left.\bar{g} \mathrm{~K} \_T s t\right)$, potassium voltage gated channel conductance ( $\bar{g} \mathrm{Kv} 3.1)$ (4), high voltage activated calcium channels conductance ( $\left.\bar{g} \mathrm{Ca} \_H V A\right)$, low voltage activated calcium channel conductance ( $\overline{\mathrm{g} C a} \_$LVA) (5), $\bar{g} \mathrm{SK}$, and gamma_CaDynamics corresponding to the percentage of free intracellular calcium levels. Further

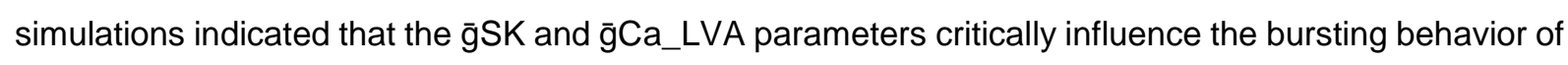
the $3 \times T$ group (Fig. 4G).

Distribution analysis of selected parameters linked to cell excitability and SK channel activity unveiled a significantly higher conductance of SK channels in the 3xTg model compared to the other groups, a smaller conductance of low voltage activated calcium (LVA) channels and an alteration of percentage of free calcium in the soma. In dendrites, LVA presented the opposite behavior, having a higher conductance in 3xTg cells compared to simulated WTs and 3xTg UCP4 neurons ( SI Appendix, Fig. S5 F). 
bioRxiv preprint doi: https://doi.org/10.1101/2022.01.25.477694; this version posted January 27, 2022. The copyright holder for this preprint (which was not certified by peer review) is the author/funder, who has granted bioRxiv a license to display the preprint in perpetuity. It is made

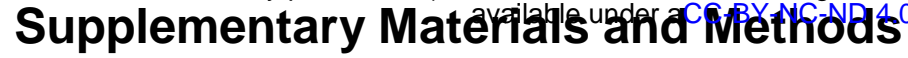

\section{Animals.}

All the experimental procedures complied with the Swiss National Institutional Guidelines on animal experimentation and were approved by the canton of Vaud Cantonal Veterinary Office Committee for Animal Experimentation (authorization \#VD3106 and VD3106_1). In all cases special attention was given to the implementation of $3 R s$, housing, environmental conditions, and analgesia to improve the animals' welfare. In all experiments, homozygous 3xTg mice (The Jackson Laboratory, MMRRC stock \#34830, RRID:MMRRC_034830-JAX) (6) adult male mice 7-10 months old were used and compared to age-and sex-matched WT (B6129SF2/J) mice, which are derived from the original mouse line with the same genetic background. We choose to work with males to avoid possible additional factors impacting $A D$, such as hormones affecting females (7). In addition, males present at early stage equal metabolic alterations as females (8), rendering them suitable for our investigations.

\section{Viral vector construction.}

AAV plasmid pAAV-GfaABC(1)D-eGFP containing the minimal GFAP promoter (GfaABC(1)D) and the enhanced Green Fluorescent Protein (eGFP) between AAV inverted terminal repeats (ITRs) sequences was kindly provided by Dr. Bernard Schneider (Bertarelli Foundation Gene Therapy Core Facility, EPFL, Switzerland) and Dr. Sylvain Lengacher (GliaFarm SA, Campus Biotech, Switzerland) with the previously described DNA sequence (9). A de novo synthesized mCherry-P2A-UCP4-HAHAHA-WPRE (GeneScript, New Jersey, USA) with the previously described mCherry (10) and UCP4 (11) linked in frame with a $2 A$ self-cleaving peptide sequence (P2A) was cloned into pAAV-GfaABC(1)D-eGFP, removing eGFP genes to obtain pAAV-GfaABC(1)D-mCherry-P2A-UCP4-HAHAHA-WPRE. PAAVGfaABC(1)D-mCherry was obtained by cloning mCherry gene from pAAV-GFAP-mCherry (12) in pAAVGfaABC(1)D-mCherry-P2A-UCP4-HAHAHA-WPRE, removing mCherry-P2A-UCP4-HAHAHA genes. PAAV-GFaABC(1)D-UCP4-HAHAHA-WPRE was obtained by cloning UCP4-HAHAHA from pIRESUCP4-HAHAHA (11) into pAAV-GfaABC(1)D-mCherry-P2A-UCP4-HAHAHA-WPRE, removing mCherry-P2A-UCP4-HAHAHA. AAV virus particles were generated as described by Grieger et al. (13) at a titer ranging from $110^{12}$ to $110^{13}(\mathrm{Vg} / \mathrm{ml})$, based on a recombinant genome containing the AAV2 ITRs and pseudotyped with an AAV capsid derived from serotype 9.

\section{Viral vector delivery and surgery.}

An initial dose of analgesic (Carprofen i.p. $5 \mathrm{mg} / \mathrm{kg}$ ) was administered subcutaneously to the 2-monthsold mice before surgeries. Mice were anesthetized with isoflurane and placed on a heating blanket to maintain the body temperature at $37^{\circ} \mathrm{C}$. The animal was head-fixed on a stereotaxic head frame apparatus (Stoelting, Wood Dale, IL). A small incision was made on the skin and the bone exposed at the desired injection site. Viruses were injected with a thin glass pipette pulled on a vertical puller (Narishige, Tokyo, Japan). WT and 3xTg-AD mice were injected bilaterally in two sites in the hippocampus (site 1. Antero-posterior: $-1 \mathrm{~mm}$, medio-lateral: $\pm 1.46 \mathrm{~mm}$, ventral: -1.5 from bregma; site 2. Antero-posterior: $-1.56 \mathrm{~mm}$, medio-lateral: $\pm 2.15 \mathrm{~mm}$, ventral: -1.5 from bregma) with a virus encoding 
bioRxiv preprint doi: https://doi.org/10.1101/2022.01.25.477694; this version posted January 27, 2022. The copyright holder for this preprint (which was not certified by peer review) is the author/funder, who has granted bioRxiv a license to display the preprint in perpetuity. It is made

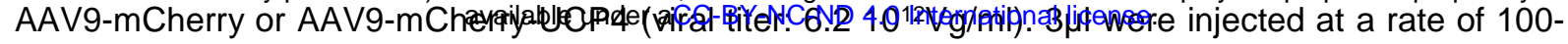

$200 \mathrm{nl} / \mathrm{min}$ in each injection site.

\section{In vivo MRI.}

MRI experiments were performed on a Bruker 3 Tesla small-bore scanner (BioSpec 30/18). A radiofrequency (RF) transmit-receive volume coil (Bruker BioSpin MRI, Ettlingen, Germany) with 82mm inner diameter and with active detuning was used for excitation, while a receive-only $2 \times 2$ channel mouse brain surface array coil (Bruker BioSpin MRI, Ettlingen, Germany) was used for signal detection. Animals were imaged six months after viral injections. They were isoflurane anesthetized during the whole imaging session. The respiration rate was monitored through a respiration pad (SA Instruments, Stony Brook, NY, USA) placed against the mouse abdomen. The body temperature was monitored with a probe placed against the mouse flank and maintained at $37 \pm 0.5^{\circ} \mathrm{C}$ using a tubing system with circulating hot water. A fast low angle shot (FLASH) MRI pulse sequence with the following parameters: repetition time $(T R)=100 \mathrm{~ms}$, echo time $(T E)=7 \mathrm{~ms}$, RF excitation angle $\alpha=40^{\circ}$, field of view $(F O V)=30 \times 30 \mathrm{~mm}$, matrix size $(M S)=256 \times 256$, slice thickness $(S T)=1 \mathrm{~mm}$, number of slices $=3$, number of averages $(N A)=2$, acquisition time $=32 \mathrm{~s}$, was used to acquire 3-plane localizer images. Then, turbo spin echo (TSE) T1weighted images with the following parameters: $T R=3300 \mathrm{~ms}, T E=60 \mathrm{~ms}$, echo spacing $=12 \mathrm{~ms}$, echo train length $(E T L)=12, F O V=20 \times 20 \mathrm{~mm}, M S=128 \times 128, S T=0.5 \mathrm{~mm}$, number of slices $=19, N A=24$, acquisition time $=13 \mathrm{~min} 12 \mathrm{~s}$, were acquired to visualize the hippocampus. MR image analysis was performed with Matlab. Hippocampi volumes were measured from multiple ROIs manually drawn by a single blinded operator on the sagittal plane.

\section{Immunohistochemistry and confocal microscopy.}

For virus testing, 4 weeks after injections, adult mice from both genotypes were transcardially perfused with cold 4\% PFA (pH 7.4). Sagittal sections $(30 \mu \mathrm{m})$ were obtained using a vibratome (VT $1000 \mathrm{~S}$,

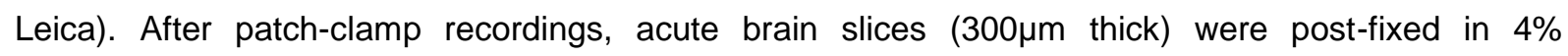
paraformaldehyde (PFA) for $>24 \mathrm{~h}$. Then, the same procedure after both experiments was performed for immunostaining: slices were first washed with PBS and permeabilized with $1 \%$ Triton $+1 \mathrm{~g} / \mathrm{l}$ azide. Nonspecific binding was blocked with PBS containing $2 \%$ normal horse serum. Samples were then incubated with the primary antibodies in blocking solution during 5 days at $4^{\circ} \mathrm{C}$ : mouse anti-GFAP (G3893, Sigma, 1:300), mouse anti-HSP70 (MA3-028, Invitrogen, 1:500) and rabbit anti-HA (ab9110, Abcam, 1:200). Floating sections were incubated with respective secondary antibodies Alexa Fluor-488 goat anti-mouse (Invitrogen, 1:200), IRDye 680RD goat anti-rabbit (LI-COR, 1:200) and streptavidin Alexa Fluor 647 (Invitrogen, 1:500) overnight at $4^{\circ} \mathrm{C}$. Finally, slices were incubated with DAPI (ThermoFisher, 1:1000) and mounted for imaging performed using Zeiss LSM780 and Zeiss LSM900 confocal microscopes.

\section{Metabolomic Profiling.}

Sample collection for metabolomic profiling. Focused Beam Microwave Irradiation was selected as euthanasia method as it optimally preserves metabolite levels after death by instantly inactivating enzymes of cellular metabolism (14). Mice were first anesthetized with a mixture of Medetomidine $(0.5 \mathrm{mg} / \mathrm{kg})$, Fentanyl $(0.05 \mathrm{mg} / \mathrm{kg})$ et Midazolam $(5 \mathrm{mg} / \mathrm{kg})$ through i.p. injection. They were then swiftly 
bioRxiv preprint doi: https://doi.org/10.1101/2022.01.25.477694; this version posted January 27, 2022. The copyright holder for this preprint (which was not certified by peer review) is the author/funder, who has granted bioRxiv a license to display the preprint in perpetuity. It is made

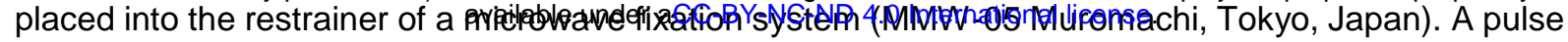
of $4 \mathrm{~kW}$ with $0.6 \mathrm{sec}$ exposure was applied to cause the immediate death. Mice were then rapidly decapitated, hippocampi dissected out, individually placed in plastic tubes, and stored on dry ice before tissue processing.

Metabolite extraction. Mouse hippocampus samples were pooled according to genotype and treatment. Samples were extracted by the addition of $\mathrm{MeOH}: \mathrm{H} 2 \mathrm{O}(4: 1)(800 \mu \mathrm{L})$. This solution containing hippocampal samples was further homogenized in the Cryolys Precellys 24 sample Homogenizer (2x20sec at 10000rpm, Bertin Technologies, Rockville, MD , US) with ceramic beads. The bead beater was air-cooled down at a flow rate of $110 \mathrm{~L} / \mathrm{min}$ at 6 bar. Homogenized extracts were centrifuged for $15 \mathrm{~min}$ at $4000 \mathrm{~g}$ at $4^{\circ} \mathrm{C}$ (Hermle, Gosheim, Germany) and the resulting supernatants $(100 \mu \mathrm{L})$ were collected and evaporated to dryness in a vacuum concentrator (LabConco, Missouri, US). Dried sample extracts were re-suspended in $\mathrm{MeOH}: \mathrm{H}_{2} \mathrm{O}(4: 1, \mathrm{v} / \mathrm{v})$ prior to LC-MS/MS analysis according to the total protein content (quantified using BCA protein Assay Kit (Thermo Scientific, Masschusetts, USA) .

Multiple pathway targeted LC-MS/MS analysis. Tissue extracts were analyzed by Hydrophilic Interaction Liquid Chromatography coupled to tandem mass spectrometry (HILIC - MS/MS) in both positive and negative ionization modes using a 6495 triple quadrupole system (QqQ) interfaced with 1290 UHPLC system (Agilent Technologies). In positive mode, the chromatographic separation was carried out in an Acquity BEH Amide, $1.7 \mu \mathrm{m}, 100 \mathrm{~mm} \times 2.1 \mathrm{~mm}$ I.D. column (Waters, Massachusetts, US). Mobile phase was composed of $A=20 \mathrm{mM}$ ammonium formate and $0.1 \% \mathrm{FA}$ in water and $\mathrm{B}=0.1 \%$ formic acid in ACN. The linear gradient elution from 95\% B (0-1.5min) down to $45 \%$ B was applied (1.5min$7 \mathrm{~min}$ ) and these conditions were held for $2 \mathrm{~min}$. Then initial chromatographic condition were maintained as a post-run during $5 \mathrm{~min}$ for column re-equilibration. The flow rate was $400 \mu \mathrm{l} / \mathrm{min}$, column temperature $25^{\circ} \mathrm{C}$ and sample injection volume $2 \mu \mathrm{l}$. In negative mode, a SeQuant ZIC-pHILIC (100mm, 2.1mm I.D. and $5 \mu \mathrm{m}$ particle size, Merck, Damstadt, Germany) column was used. The mobile phase was composed of $\mathrm{A}=20 \mathrm{mM}$ ammonium Acetate and $20 \mathrm{mM} \mathrm{NH} 4 \mathrm{OH}$ in water at $\mathrm{pH} 9.7$ and $\mathrm{B}=100 \% \mathrm{ACN}$. The linear gradient elution from $90 \%(0-1.5 \mathrm{~min})$ to $50 \% \mathrm{~B}(8-11 \mathrm{~min})$ down to $45 \% \mathrm{~B}(12-15 \mathrm{~min})$. Finally, the initial chromatographic conditions were established as a post-run during $9 \mathrm{~min}$ for column re-equilibration. The flow rate was $300 \mu \mathrm{l} / \mathrm{min}$, column temperature $30^{\circ} \mathrm{C}$ and sample injection volume $2 \mu \mathrm{l}$. ESI source conditions were set as follows: dry gas temperature $290^{\circ} \mathrm{C}$ and flow $14 \mathrm{~L} / \mathrm{min}$, sheath gas temperature $350^{\circ} \mathrm{C}$, nebulizer $45 \mathrm{psi}$, and flow $12 \mathrm{l} / \mathrm{min}$, nozzle voltage $0 \mathrm{~V}$, and capillary voltage $+/-2000 \mathrm{~V}$. Dynamic Multiple Reaction Monitoring (dMRM) was used as acquisition mode with a total cycle time of $600 \mathrm{~ms}$. Optimized collision energies for each metabolite were applied.

Pooled QC sample solution (representative of the entire sample set) was analyzed periodically (every 5 samples) throughout the overall analytical run. In addition, a series of diluted quality controls (dQC) were prepared by dilution with methanol: 100\% QC, 50\% QC, 25\% QC, 12.5\% QC and 6.25\% QC and analyzed at the beginning and at the end of the sample batch.

Data (pre)processing and quality assessment. Raw LC-MS/MS data was processed using the Agilent Quantitative analysis software (version B.07.00, MassHunter Agilent technologies). Relative quantification of metabolites was based on EIC (Extracted lon Chromatogram) areas for the monitored 
bioRxiv preprint doi: https://doi.org/10.1101/2022.01.25.477694; this version posted January 27, 2022. The copyright holder for this preprint (which was not certified by peer review) is the author/funder, who has granted bioRxiv a license to display the preprint in perpetuity. It is made

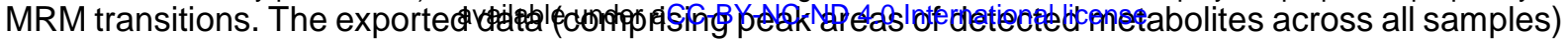
were corrected for the signal intensity drift (over time) using LOWESS/Spline algorithm. The metabolite features represented by "not-well behaving" peaks (CV ( $Q$ C peaks) $>30 \% \& R^{2}$ ( $Q C$ dilution curve) were discarded from further statistical analysis.

Statistical data analysis. Two-way Anova (on log10 transformed peak abundances) were used to test the significance of metabolite changes between different groups of samples (3xTg-UPC4, 3xTg, WTUPC4 and WT) with an arbitrary level of significance, $p$-value $=0.05$ (and adjusted $p$-value corrected for multiple testing with Benjamini-Hochberg method).

\section{Acute slice preparation, patch clamp recordings.}

Mice were deeply anaesthetized with ketamine $(100 \mathrm{mg} / \mathrm{kg}$, ip) and xylazine $(10 \mathrm{mg} / \mathrm{kg}$, ip) and then transcardially perfused with $10-20 \mathrm{ml}$ of ice-cold NMDG artificial cerebrospinal fluid (ACSF) containing (mM): $93 \mathrm{NMDG}, 2.5 \mathrm{KCl}, 1.2 \mathrm{NaH}_{2} \mathrm{PO}_{4}, 30 \mathrm{NaHCO}_{3}, 20$ HEPES, 25 glucose, 5 ascorbic acid, 2 thiourea, 3 sodium pyruvate, $12 \mathrm{~N}$-acetyl-L-cysteine, $10 \mathrm{MgSO}_{4}, 0.5 \mathrm{CaCl}_{2}$ (15), pH 7.3, equilibrated with $95 \% \mathrm{O}_{2}$ and $5 \% \mathrm{CO}_{2}$. Next, mice were decapitated and brains quickly removed and placed into the same NMDG ACSF. 300 $\mu \mathrm{m}$ thick sagittal acute slices were prepared using a vibratome (VT1000S, Leica) in the same ice-cold ACSF. Slices were incubated in ACSF containing (mM): $92 \mathrm{NaCl}, 2.5 \mathrm{KCl}$, $1.2 \mathrm{NaH}_{2} \mathrm{PO}_{4}, 30 \mathrm{NaHCO}_{3}, 20 \mathrm{HEPES}, 25$ glucose, 5 ascorbic acid, 2 thiourea, 3 sodium pyruvate, 12 $\mathrm{N}$-acetyl-L-cysteine, $2 \mathrm{MgSO}_{4}, 2 \mathrm{CaCl}_{2}$, for $20 \mathrm{~min}$ at $34^{\circ} \mathrm{C}$ and then kept at room temperature for at least $20 \mathrm{~min}$ before being transferred to the recording chamber. Slices were held down by a metal harp and superfused by oxygenated ACSF containing (in mM): $119 \mathrm{NaCl}, 2.5 \mathrm{KCl}, 1 \mathrm{NaH}_{2} \mathrm{PO}_{4}, 26.2 \mathrm{NaHCO}_{3}, 11$ glucose, $1.3 \mathrm{MgSO}_{4}, 2.5 \mathrm{CaCl}_{2}$ at $32^{\circ} \mathrm{C}$. Recordings were made under a Zeiss LSM510 Meta upright microscope equipped with infrared DIC using a 40X water-dipping objective lens (Carl Zeiss). Wholecell patch-clamp of subicular neurons was obtained with borosilicate glass pipettes (6-8 $\mathrm{M} \Omega$ resistance). The patch-clamp intracellular solution contained (in $\mathrm{mM}$ ): $130 \mathrm{~K}$-gluconate, $5 \mathrm{NaCl}, 5 \mathrm{KCl}, 1 \mathrm{MgCl}_{2}, 0.1$ EGTA, $0.025 \mathrm{CaCl}_{2}$, 10 HEPES, 4 glucose, $5 \mathrm{Na}$ phosphocreatine, $4 \mathrm{Mg}$-ATP, $0.3 \mathrm{Na}$-GTP, pH 7.3 with $\mathrm{KOH}, 290$ mOsm. $0.1 \%$ biocytin was added to the pipette solution to further visualize the neurons patched. Recordings were obtained in current clamp configuration using a Multiclamp 700B amplifier (Molecular Devices). Data were acquired at $10 \mathrm{kHz}$ and filtered at $2 \mathrm{kHz}$ (Digidata 1440 analog-to-digital converter), controlled with the PCLAMP 10 software. Experiments were discarded if the access resistance, monitored by $-5 \mathrm{mV}$ steps $(0.1 \mathrm{~Hz})$, varied by more than $20 \%$. Current steps (30pA increments) of $320 \mathrm{~ms}$ duration were injected to measure burst frequency and of $2000 \mathrm{~ms}$ for assessing AHPs. In a subset of experiments, the SK channel enhancer ns-309 ( $5 \mu \mathrm{M}$, Sigma-Aldrich) was bath applied $10 \mathrm{~min}$ before repeating the same measurements. The amplitude of AHPs and burst frequency were quantified using the Clampfit software.

\section{Sholl and morphometric analysis.}

Morphological analyses of neurons were made from $80-100 \mu \mathrm{m}$ confocal image stacks acquired using a 20X 0.8NA Plan Apochromat objective lens (Carl Zeiss) on a Zeiss LSM900 microscope, and 3Dreconstructed using Imaris Filament Tracer (Bitplane AG, Switzerland). Reconstructions were performed in a blinded experimental design. For each reconstructed 3D morphology, we performed a Sholl and 
bioRxiv preprint doi: https://doi.org/10.1101/2022.01.25.477694; this version posted January 27, 2022. The copyright holder for this preprint (which was not certified by peer review) is the author/funder, who has granted bioRxiv a license to display the preprint in perpetuity. It is made

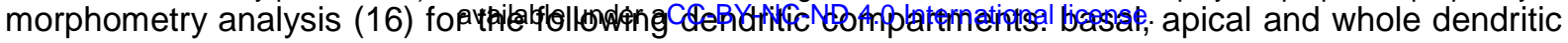
tree. For Sholl analysis, concentric rings were placed on the cell centered at the soma. The radius of the ring was incremented by $5 \mu \mathrm{m}$ steps until dendrites were completely covered. Number of dendritic crossings with the ring were calculated for each ring radius. For morphometry analysis, we measured the number and length of dendritic branches, using open source Python package NeuroM (https://github.com/BlueBrain/NeuroM).

\section{Electrophysiological modeling.}

The features from voltage recordings were extracted using BluePyEfe package (https://github.com/BlueBrain/BluePyEfe) and Electrophys Feature Extraction Library (eFEL, https://github.com/BlueBrain/BluePyEfe). For each cell a rheobase was calculated. Features were extracted from the recordings of a chosen fraction from rheobase and averaged among the sweeps and cells. Number of spikes, inter spikes intervals, voltage base, AHP after each spike, time of AHP relative to AP peak, first second and last AP amplitudes, voltage at the beginning of AP, slope and coefficient of variation $(\mathrm{CV})$ of inter spike intervals, AP width and amplitude of the AHP after the stimuli were extracted from voltage recordings in response to depolarizing steps (for $200 \mathrm{~ms}$ step we considered step amplitudes of 200, 300 and 500\% from the rheobase, for 2000ms step -100, 150, 300 and 400\% from the rheobase). Base voltage, voltage deflections, and voltage at the steady state after the stimuli were extracted for the hyperpolarization step.

The neuronal model consisted of the 3D morphological reconstruction and a number of ionic mechanisms. The following mechanisms were considered: calcium high voltage activated channels (17), calcium low voltage activated channels (18), persistent and transient potassium currents (19), SK type calcium activated potassium current (20), Kv1.3 potassium current (21), lh current (22), transient and persistent sodium currents (23) and intracellular calcium dynamic (24). The models of the mechanism are adapted Markram et al. (25). The maximal conductance of the aforementioned mechanisms, leak conductance, membrane capacitance, leak reversal potential and exponential decays of sodium and Ih current were set as free parameters, while axial resistance was set to $100 \mathrm{Ohm} / \mathrm{cm}$. Initial simulation voltage was set to $-80 \mathrm{mV}$, simulation temperature to $34^{\circ} \mathrm{C}$, and equilibrium potentials for sodium and potassium to $50 \mathrm{mV}$ and $-90 \mathrm{mV}$, respectively. All parameters of ionic mechanisms, such as membrane capacitance and intracellular calcium dynamics were specifically optimized for soma and dendrite, and axon. EPSP stimuli (25) were placed in the middle of the morphological compartment.

The models were implemented in NEURON (26), and optimization of ionic maximal conductance and calcium dynamics parameters were performed with Indicator Based Evolutionary Algorithm (IBEA) using BluePyOpt python package (27). A successful model was considered to have all feature errors less than 5 SD of experimental mean feature. We generated 100 models for each group of neurons, using different seed values, to diversify the output. Several models with the best standard scores were chosen (WT n=5, WT UCP4 n=5, 3xTg n=7, 3xTg-AD UPC4 n=5).

\section{Behavioral assay.}

Novel Object Location testing was performed using an arena consisting of a custom built graybox $(\mathrm{W}=\mathrm{L}=\mathrm{H}: 45 \mathrm{~cm})$. The light source was placed below the horizon of the arena and provided $45 \mathrm{Lux}$, 
bioRxiv preprint doi: https://doi.org/10.1101/2022.01.25.477694; this version posted January 27, 2022. The copyright holder for this preprint (which was not certified by peer review) is the author/funder, who has granted bioRxiv a license to display the preprint in perpetuity. It is made

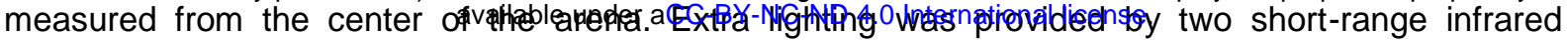
illuminators (Raytec, Raymax RM25) mounted on the room ceiling. The objects were geometric shapes $(\mathrm{W}=\mathrm{L}: 6.5 \mathrm{~cm}$ and $\mathrm{H}: 4 \mathrm{~cm})$ built with Lego Duplo ${ }^{\mathrm{TM}}$. The color of the objects was uniform blue, red, or green and it was confounded across animals and groups. Mice were brought to the experimental room 30 min prior to the beginning of the behavioral assay. The experiment consisted of 6 trials (duration: 5min, inter trial interval: 3min) run in one single day (between 10:00 and 16:00). In trial one, the habituation trial, each mouse was individually placed in the empty arena. During trials 2 through 4, the exploration trials, three objects were placed according to the pattern depicted in Fig. 5A (middle). These trials were used as an internal control to let the animals familiarize with the objects and to identify potential bias towards one object over the others. Indeed, mice that showed more than $50 \%$ of contacts toward one particular object in at least two sessions were excluded from further analyses. In trials 5 and 6 , the novel object location (NOL) trials, one object was moved (object A) by $20 \mathrm{~cm}$ to define the pattern depicted in Fig. 5A (right). An overhead camera (Basler, acA1300-60gm) driven by the Ethovision software (Noldus Information Technology) automatically tracked the mouse position across trials. Traveled distance was measured throughout the trials. An exploratory event directed toward the object was defined as the entrance of the mouse nose within a zone of $2 \mathrm{~cm}$ from the object border; the object exploration zone (OEZ). The total number of entrances in the OEZ was used to calculate a preference index throughout sessions 2 to 6 . Mice that did not show any contact with the objects during sessions 2 through 4 or that had a strong preference for one of the 3 objects (defined as $>50 \%$ contacts directed toward one of the objects during 2 out of 3 trials of the exploratory trial) were excluded from subsequent analyses. Following these criteria definitions, a total of 26 subjects were excluded from the analyses. In Fig. S5 A one outlier in the 3xTg-AD UCP4 group was detected but was not excluded from further analyses. Reaction to spatial change in the NOL trials was defined as the total number of contacts with object $A$ across trials 5 and 6 and was expressed as \% contacts over the total number of contacts with the 3 objects. The graphs report the \% contacts with object A compared to the average \% contacts with the two non-displaced objects ( $B$ and $C$ ). Behavior analyses were performed by an experimenter blinded to the treatment allocation of each experimental group. 


\section{Supplementary Figưrèils}
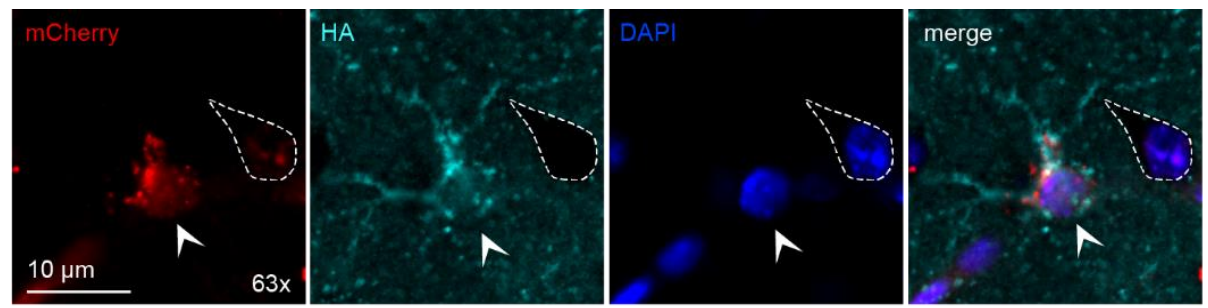

Fig. S1. UCP4 expression at 4 months post injection. Confocal image of an astrocyte and a neuron in the CA1 region (highlighted with a white arrow and a white dashed line, respectively) showing HA-tag (cyan) fluorescent immunostaining and mCherry (red) plus DAPI (blue) revealing specific HA-tag expression in astrocyte only at 4 months in WT mice. 
bioRxiv preprint doi: https://doi.org/10.1101/2022.01.25.477694; this version posted January 27, 2022. The copyright holder for this preprint (which was not certified by peer review) is the author/funder, who has granted bioRxiv a license to display the preprint in perpetuity. It is made

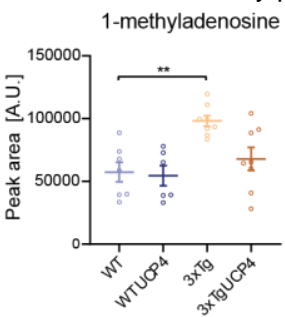

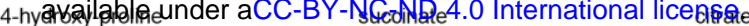
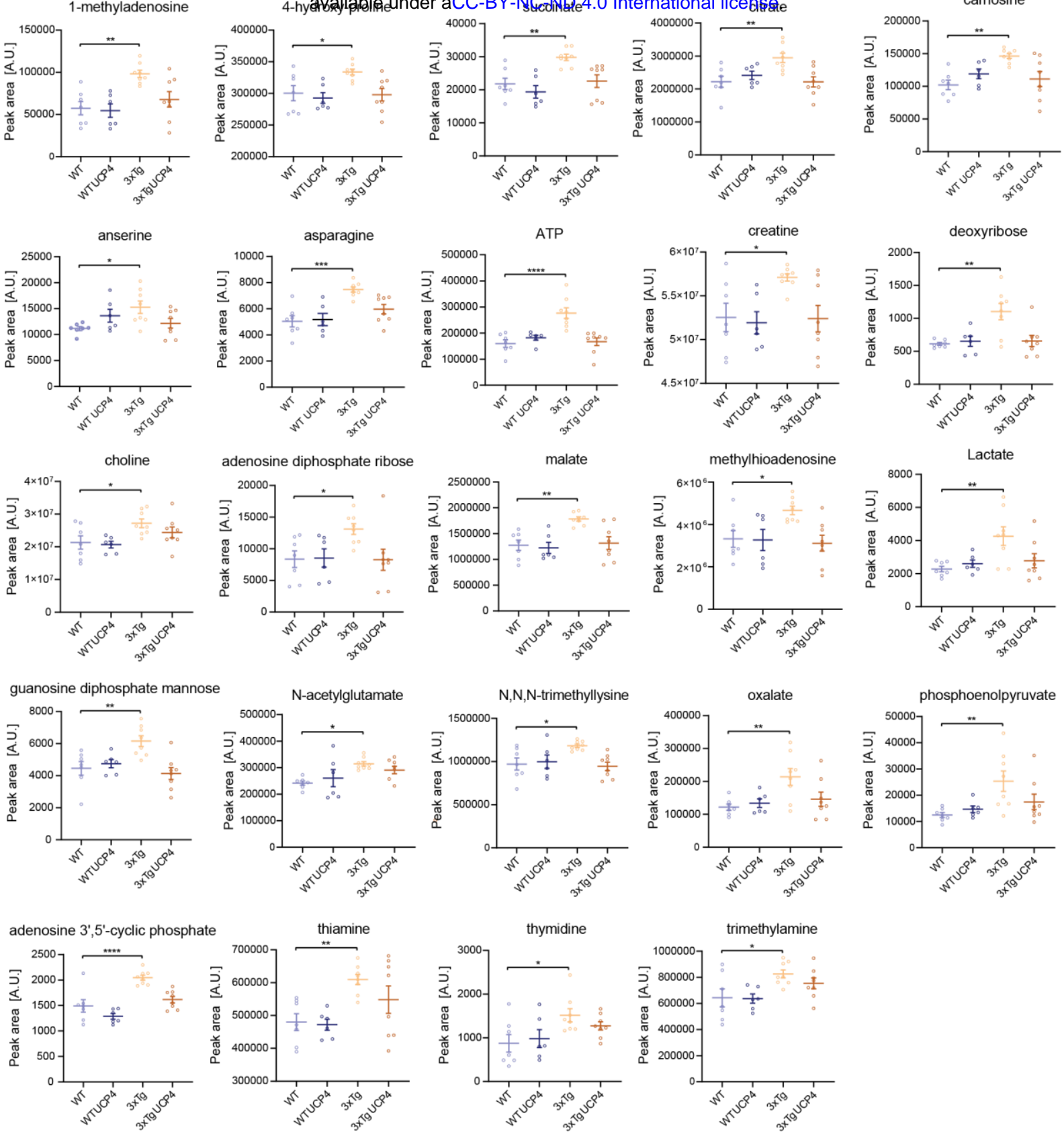

Fig. S2. Metabolites that are altered in 3xTg mice but which are maintained to WT levels by overexpression of UCP4. Plots of selected metabolites comparing hippocampal levels in WT $(n=7)$, WT UCP4 ( $n=6)$, 3xTg-AD ( $n=8)$, and 3xTg-AD UCP4 $(n=8)$ mice. Each dot represents a different specimen. Two-way ANOVA followed by Tukey's post hoc test was performed. ${ }^{*} P<0.05 ;{ }^{* \star} P<0.01 ;{ }^{* * \star} P$ $<0.001 ;{ }^{* * \star *} P<0.0001$. 
bioRxiv preprint doi: https://doi.org/10.1101/2022.01.25.477694; this version posted January 27, 2022. The copyright holder for this preprint (which was not certified by peer review) is the author/funder, who has granted bioRxiv a license to display the preprint in perpetuity. It is made A

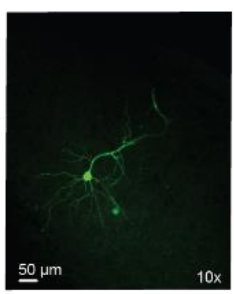
available under aCC-BY-NC-ND 4.0 International license.
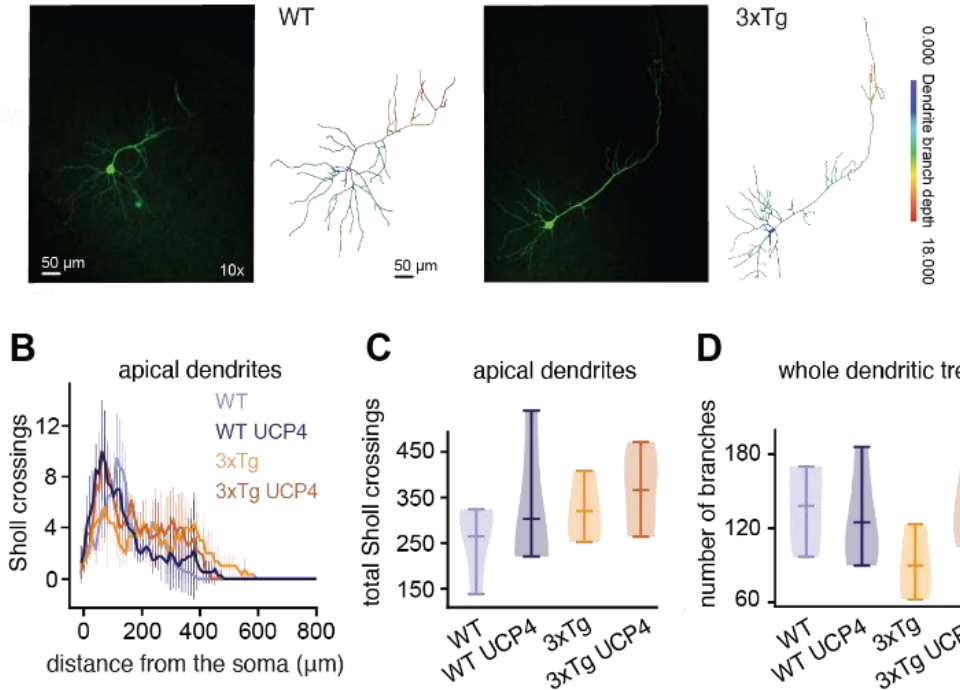

Fig. S3. Sholl profiles and morphometrics of apical dendrites. (A) Representative confocal images of WT (left) and 3xTg (right) subicular neurons filled with biocytin. The respective 3D reconstructions are shown with color-coded dendrite branch depth. (B) Sholl profil for each group, calculated using 5 $\mu \mathrm{m}$ increments (WT: $\mathrm{n}=4$, WT UCP4: $n=4$, 3xTg: $n=3$, 3xTg UCP4: $n=5$ ). (C) Total number of Sholl crossing of apical dendrites for each group. No significant statistical difference was found between the groups. (D) Total number of dendritic branches for each group, calculated for each morphology. No significant statistical difference was found between the groups. Bars on violin plots are mean $\pm \mathrm{Cl}$. 
bioRxiv preprint doi: https://doi.org/10.1101/2022.01.25.477694; this version posted January 27, 2022. The copyright holder for this preprint (which was not certified by peer review) is the author/funder, who has granted bioRxiv a license to display the preprint in perpetuity. It is made

A

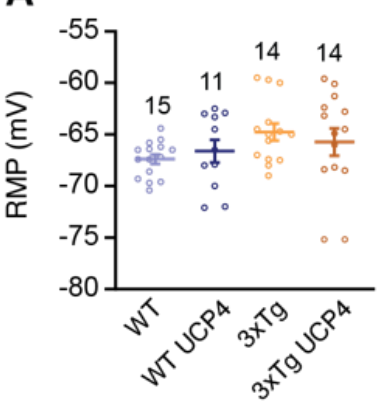

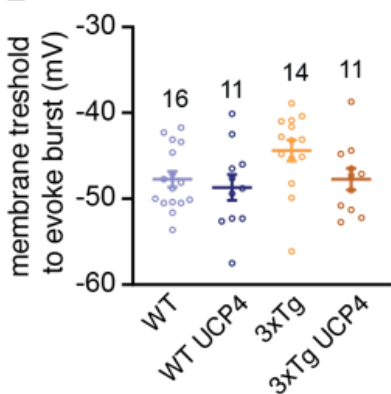

E

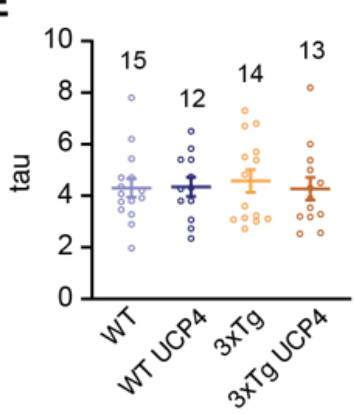

$\mathbf{F}$

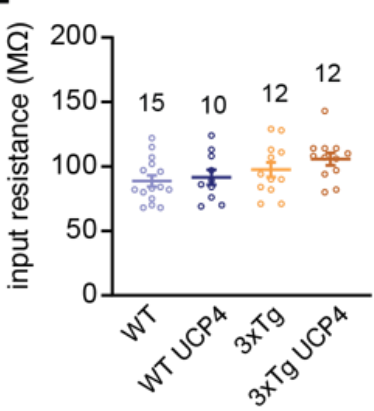

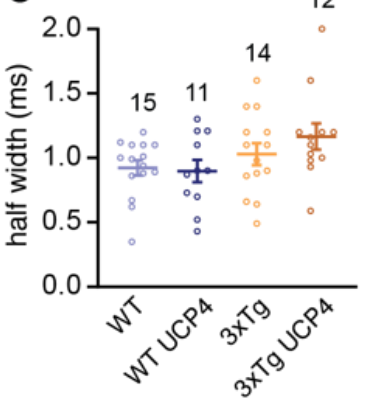

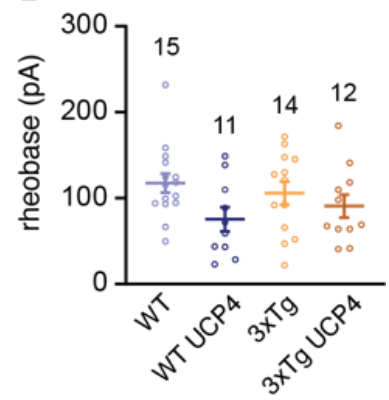

G

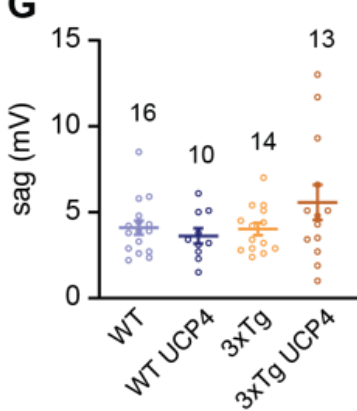

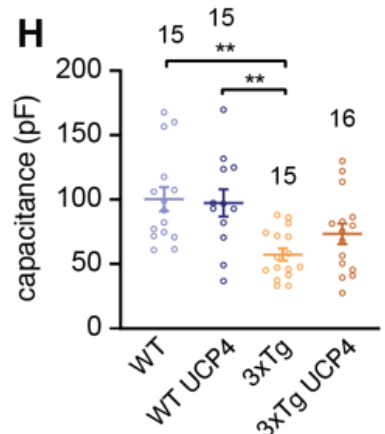

Fig.S4. Intrinsic electrophysiological properties of subicular neurons are not altered by viral constructs. Patch-clamp recordings of subicular neurons show no differences of intracellular properties, namely (A) resting membrane potential (RMP), (B) threshold to evoke burst, (C) AP half width, (D) rheobase, (e) decay (tau), (F) input resistance, and (G) sag among groups, except for $(\mathbf{H})$ capacitance. The numbers above each data set represent the number of neurons patched. In $(\mathbf{H})$ oneway ANOVA followed by Tukey's post hoc test was performed. Error bars are mean \pm SEM. ${ }^{* *} P<0.01$. 
bioRxiv preprint doi: https://doi.org/10.1101/2022.01.25.477694; this version posted January 27, 2022. The copyright holder for this preprint (which was not certified by peer review) is the author/funder, who has granted bioRxiv a license to display the preprint in perpetuity. It is made
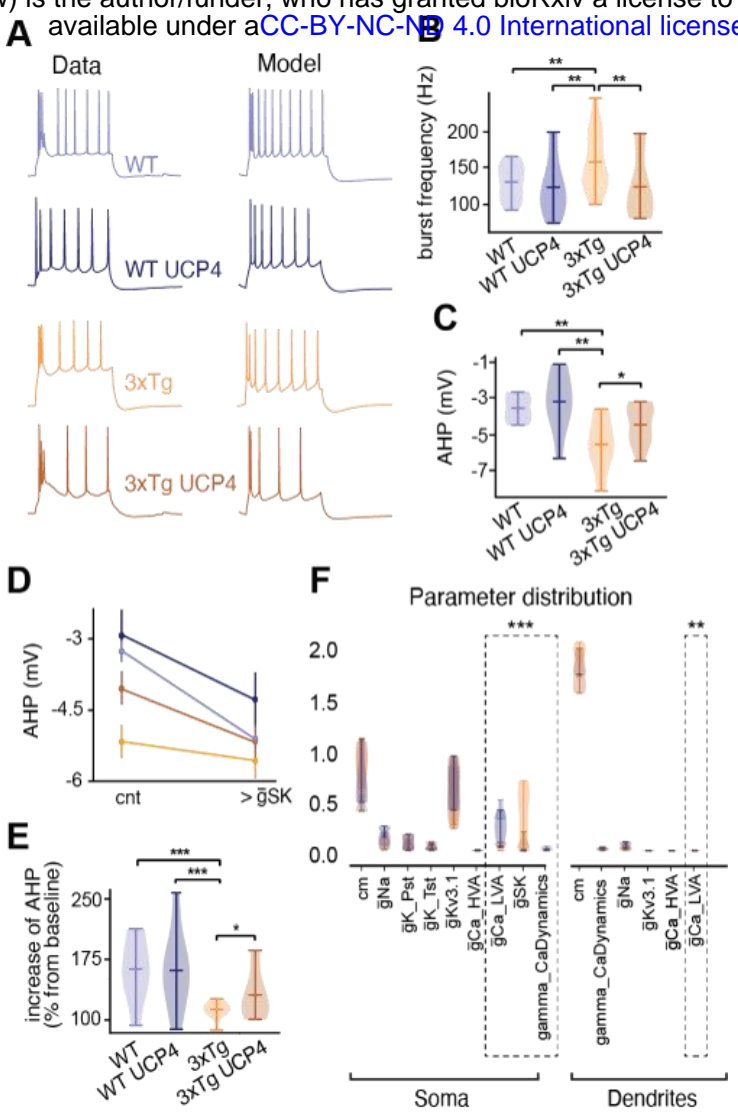

Fig. S5. Generation and validation of subicular neuron models. (A) Example traces of current clamp recordings (Data) and corresponding traces from neuronal models (Model). (B) Violin plot of burst frequencies in responses to simulated 200, 300 and 450pA current injections. Several models were considered for each group (WT: $n=5$, WT UCP4: $n=5,3 x T g: n=7,3 x T g$ UPC4: $n=5$ ). (C) Violin plot of AHP in responses to pooled 200 and 450pA current injections. (D) AHP in control condition (cnt, same as in C), compared to a manually increased SK conductance ( $>\bar{g} S K)$, across pooled models' responses to 200 and 450pA current injections. (E) Violin plot of AHP with increased ḡSK shown as percentage of control AHP amplitude. (F) Distribution of model parameters compared across groups of models (same color scheme as in $(\mathbf{A}))$. Those include unitary capacitance $(\mathrm{cm})$, sodium channel conductance $(\overline{\mathrm{g}} \mathrm{Na})$, persistent potassium conductance ( $\left.\bar{g} \mathrm{~K} \_P s t\right)$, transient potassium conductance ( $\left.\bar{g} \mathrm{~K} \_\mathrm{Tst}\right)$, potassium voltage gated channel conductance (ḡKv3.1) (4), high voltage activated calcium channels conductance ( $\bar{g} \mathrm{Ca}$ _HVA), low voltage activated calcium channel conductance ( $\bar{g} \mathrm{Ca}$ _LVA) (5), $\bar{g} S K$, and gamma_CaDynamics. Significant statistical difference between groups are highlighted by the dashed line rectangles. Scale on the left: absolute value. Bars on violin plots are mean \pm CI. In (B), (C) and (E), nonparametric bootstrap test was performed. In (D) error bars represent mean \pm SEM. In (F), KruskalWallis followed by a post hoc Bonferroni correction test was performed. ${ }^{*} P<0.05$; ${ }^{* *} P<0.01$; ${ }^{* *} P<$ 0.001 . Bars on violin plots are mean $\pm \mathrm{Cl}$. 

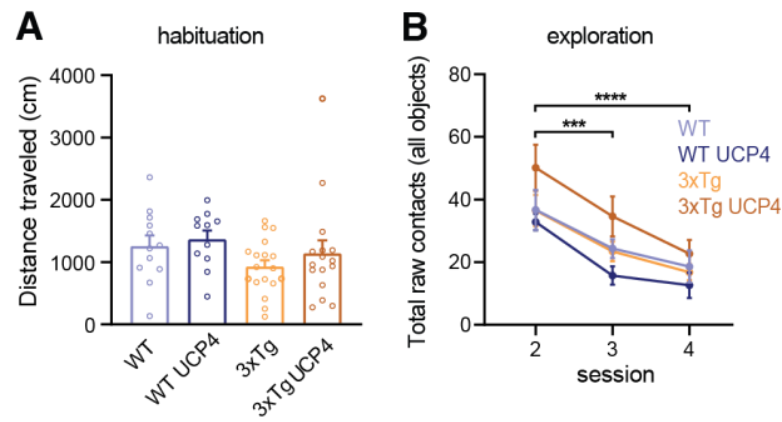

Fig. S6. Viral vectors do not affect overall locomotory behavior. (A) Distance traveled by the mice during the habituation trial. (WT: $n=12$, WT UCP4: 11, 3xTg: $n=18,3 \times T g$ UCP4: $n=16$ ). (B) Overall number of contacts with the 3 objects across sessions. Data are the average contact number with objects (same $\mathrm{n}$ per groups as in $\mathbf{A}$ ). In (A) one-way ANOVA followed by Tukey's post hoc test was performed. In (B) a two-way ANOVA followed by Tukey's post hoc test was performed, error bars are mean \pm SEM. ${ }^{* * \star} P<0.001 ;{ }^{* * *} P<0.0001$. 
bioRxiv preprint doi: https://doi.org/10.1101/2022.01.25.477694; this version posted January 27, 2022. The copyright holder for this preprint (which was not certified by peer review) is the author/funder, who has granted bioRxiv a license to display the preprint in perpetuity. It is made

\section{Supplementary Referenteres}

1. T. J. Ngo-Anh et al., SK channels and NMDA receptors form a Ca2+-mediated feedback loop in dendritic spines. Nat Neurosci 8, 642-649 (2005).

2. S. Chakroborty et al., Early presynaptic and postsynaptic calcium signaling abnormalities mask underlying synaptic depression in presymptomatic Alzheimer's disease mice. $J$ Neurosci 32, 83418353 (2012).

3. J. P. Adelman, J. Maylie, P. Sah, Small-conductance Ca2+-activated K+ channels: form and function. Annu Rev Physiol 74, 245-269 (2012).

4. B. Rudy, C. J. McBain, Kv3 channels: voltage-gated $\mathrm{K}+$ channels designed for high-frequency repetitive firing. Trends Neurosci 24, 517-526 (2001).

5. E. Perez-Reyes, Molecular physiology of low-voltage-activated t-type calcium channels. Physiol Rev 83, 117-161 (2003).

6. S. Oddo et al., Triple-transgenic model of Alzheimer's disease with plaques and tangles: intracellular Abeta and synaptic dysfunction. Neuron 39, 409-421 (2003).

7. L. K. Clinton et al., Age-dependent sexual dimorphism in cognition and stress response in the 3xTgAD mice. Neurobiol. Dis. 28, 76-82 (2007).

8. Y. Dong, G. J. Brewer, Global Metabolic Shifts in Age and Alzheimer's Disease Mouse Brains Pivot at NAD+/NADH Redox Sites. J. Alzheimers Dis. 71, 119-140 (2019).

9. E. Dirren et al., Intracerebroventricular injection of adeno-associated virus 6 and 9 vectors for cell type-specific transgene expression in the spinal cord. Hum. Gene Ther. 25, 109-120 (2014).

10. N. C. Shaner et al., Improved monomeric red, orange and yellow fluorescent proteins derived from Discosoma sp. red fluorescent protein. Nat. Biotechnol. 22, 1567-1572 (2004).

11. H. Perreten Lambert et al., Control of mitochondrial $\mathrm{pH}$ by uncoupling protein 4 in astrocytes promotes neuronal survival. J. Biol. Chem. 289, 31014-31028 (2014).

12. Y. Bernardinelli et al., Activity-dependent structural plasticity of perisynaptic astrocytic domains promotes excitatory synapse stability. Curr. Biol. 24, 1679-1688 (2014).

13. J. C. Grieger, V. W. Choi, R. J. Samulski, Production and characterization of adeno-associated viral vectors. Nat. Protoc. 1, 1412-1428 (2006).

14. T. Matsui et al., Astrocytic glycogen-derived lactate fuels the brain during exhaustive exercise to maintain endurance capacity. Proc. Natl. Acad. Sci. U.S.A. 114, 6358-6363 (2017).

15. M. Martinez-Losa et al., Nav1.1-Overexpressing Interneuron Transplants Restore Brain Rhythms and Cognition in a Mouse Model of Alzheimer's Disease. Neuron 98, 75-89.e75 (2018).

16. D. A. Sholl, Dendritic organization in the neurons of the visual and motor cortices of the cat. J. Anat. 87, 387-406 (1953).

17. I. Reuveni, A. Friedman, Y. Amitai, M. J. Gutnick, Stepwise repolarization from Ca2+ plateaus in neocortical pyramidal cells: evidence for nonhomogeneous distribution of HVA Ca2+ channels in dendrites. J. Neurosci. 13, 4609-4621 (1993).

18. R. B. Avery, D. Johnston, Multiple channel types contribute to the low-voltage-activated calcium current in hippocampal CA3 pyramidal neurons. J. Neurosci. 16, 5567-5582 (1996). 
bioRxiv preprint doi: https://doi.org/10.1101/2022.01.25.477694; this version posted January 27, 2022. The copyright holder for this preprint (which was not certified by peer review) is the author/funder, who has granted bioRxiv a license to display the preprint in perpetuity. It is made

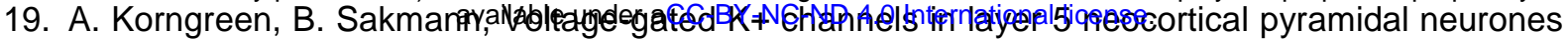
from young rats: subtypes and gradients. J. Physiol. 525 Pt 3, 621-639 (2000).

20. M. Köhler et al., Small-conductance, calcium-activated potassium channels from mammalian brain. Science 273, 1709-1714 (1996).

21. J. Rettig et al., Characterization of a Shaw-related potassium channel family in rat brain. EMBO J. 11, 2473-2486 (1992).

22. M. H. Kole, S. Hallermann, G. J. Stuart, Single Ih channels in pyramidal neuron dendrites: properties, distribution, and impact on action potential output. J. Neurosci. 26, 1677-1687 (2006).

23. C. M. Colbert, E. Pan, Ion channel properties underlying axonal action potential initiation in pyramidal neurons. Nat. Neurosci. 5, 533-538 (2002).

24. A. Destexhe, D. Contreras, T. J. Sejnowski, M. Steriade, A model of spindle rhythmicity in the isolated thalamic reticular nucleus. J. Neurophysiol. 72, 803-818 (1994).

25. H. Markram et al., Reconstruction and Simulation of Neocortical Microcircuitry. Cell 163, 456-492 (2015).

26. M. L. Hines, N. T. Carnevale, The NEURON simulation environment. Neural Comput. 9, 1179-1209 (1997).

27. W. Van Geit et al., BluePyOpt: Leveraging Open Source Software and Cloud Infrastructure to Optimise Model Parameters in Neuroscience. Front. Neuroinform. 10, 17 (2016). 